\title{
COMMUNICATION IN PATIENT CARE HANDOFFS AND IMPLICATIONS TO \\ SAFETY
}

\author{
by \\ Alicia McMullan \\ (Bachelor of Arts, Honours, Carleton University, 2007)
}

\begin{abstract}
A thesis submitted to
The Faculty of Graduate and Postdoctoral Affairs

in partial fulfillment of the requirements for the degree of

Master of Arts

in

Psychology
\end{abstract}

Carleton University

Ottawa, Canada

(C)2010 Alicia McMullan 


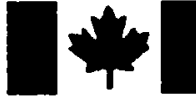

Library and Archives

Canada

Published Heritage

Branch

395 Wellington Street

Ottawa ON K1A ON4

Canada
Bibliothèque et

Archives Canada

Direction du

Patrimoine de l'édition

395 , rue Wellington

Ottawa ON K1A 0N4

Canada
Your file Votre reférence
ISBN: $978-0-494-71690-8$
Our file Notre reférence
ISBN: $978-0-494-71690-8$
NOTICE:

The author has granted a nonexclusive license allowing Library and Archives Canada to reproduce, publish, archive, preserve, conserve, communicate to the public by telecommunication or on the Internet, loan, distribute and sell theses worldwide, for commercial or noncommercial purposes, in microform, paper, electronic and/or any other formats.

The author retains copyright ownership and moral rights in this thesis. Neither the thesis nor substantial extracts from it may be printed or otherwise reproduced without the author's permission.
AVIS:

L'auteur a accordé une licence non exclusive permettant à la Bibliothèque et Archives Canada de reproduire, publier, archiver, sauvegarder, conserver, transmettre au public par télécommunication ou par l'Internet, prêter, distribuer et vendre des thèses partout dans le monde, à des fins commerciales ou autres, sur support microforme, papier, électronique et/ou autres formats.

L'auteur conserve la propriété du droit d'auteur et des droits moraux qui protège cette thèse. $\mathrm{Ni}$ la thèse ni des extraits substantiels de celle-ci ne doivent être imprimés ou autrement reproduits sans son autorisation.
In compliance with the Canadian Privacy Act some supporting forms may have been removed from this thesis.

While these forms may be included in the document page count, their removal does not represent any loss of content from the thesis.
Conformément à la loi canadienne sur la protection de la vie privée, quelques formulaires secondaires ont été enlevés de cette thèse.

Bien que ces formulaires aient inclus dans la pagination, il n'y aura aucun contenu manquant. 


\begin{abstract}
There are several processes in healthcare systems that do not have standardizations in place; of particular interest is the process of patient handoffs. The transfer of care from one healthcare provider to another involves detailed, high-pressure communication contexts and is often a point of information loss. The present study conducted ethnographic observations and interviews to qualitatively analyze communication behaviours and document handoff procedures in a hospital unit. Through the study of communication exchanges between multidisciplinary care providers, the researcher was able to analyze the relationships between types of information discussed, verbal behaviours, care complexity, and the use of resources. Retrospective medical information categories were the focus of verbal communications and reporting and questions were identified as the most common verbal behaviours. In the future, the results will contribute to the development of design criteria for handoff support tools and suggestions for standards of information exchange in handoffs.
\end{abstract}




\section{Acknowledgements}

First and foremost I would like to thank my advisor, Dr. Avi Parush, for the years of support and guidance and overall dedication to all of his students. Throughout my university career thus far he has donated countless hours and advice in the development of my research abilities. The skills and knowledge that I have gained will be invaluable throughout my upcoming academic endeavors. I would also like to like my committee members, especially Dr. Kathryn Momtahan and Dr. Gitte Lindgaard who have provided insightful feedback and continuous support during my studies. A special thanks to Dr. Kathryn Momtahan for her dedication and passion for research in this area, without her help this project would not have been possible. I would also like to acknowledge the support of The Canadian Patient Safety Institute's studentship in partnership with The Ottawa Hospital, especially Ms. Linda Hunter who helped to initiate the project. Finally, I would like to thank my family and friends for their continuous motivation and endless support through this entire process; without them I would not have been able to accomplish any of this. 


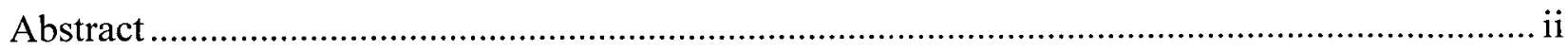

Acknowledgements.............................................................................................................

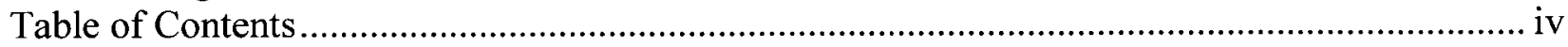

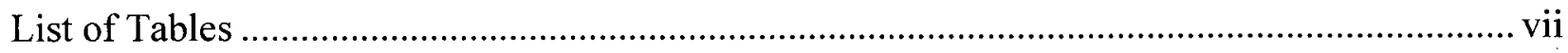

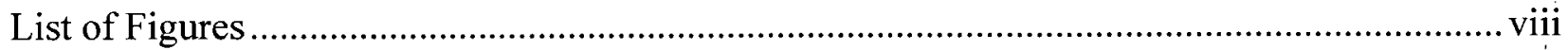

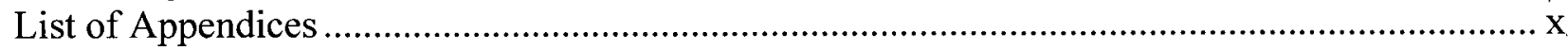

Communication in Patient Care Handoffs ............................................................................ 1

And Implications to Safety ……..................................................................................... 1

An Overview on Patient Safety.................................................................................... 2

The Importance of Patient Safety in Critical Care Environments ............................................. 4

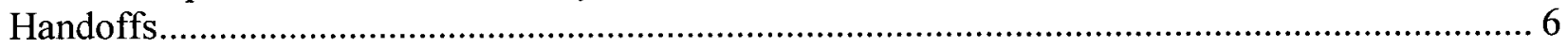

Patient Safety and Communication............................................................................... 7

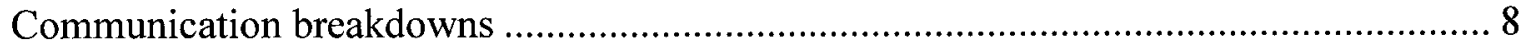

The Importance of Communication During the Patient Care Handoff.................................... 10

Multidisciplinary Communication and Care........................................................................ 13

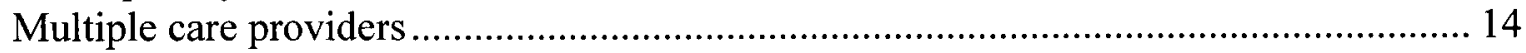

Cross-professional communication.......................................................................... 15

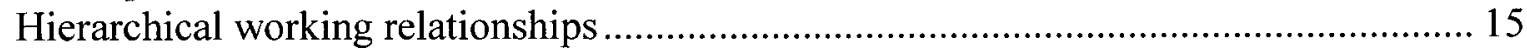

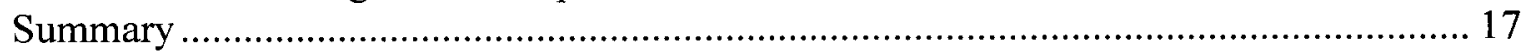

Current Methods and Modes of Communication in Healthcare ............................................ 17

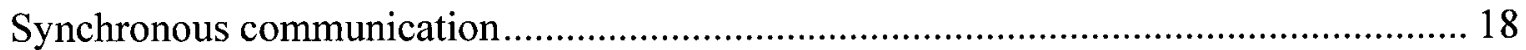

Communication problems associated with synchronous communication ......................... 20

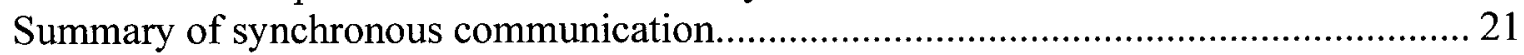

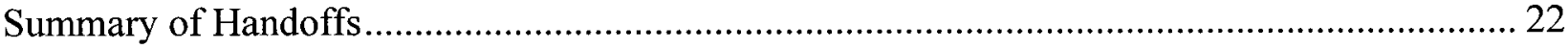

Distributed Cognition as a Theoretical Framework …………..................................................... 22

Distributed Cognition and The Patient Care Handoff.......................................................... 24

Summary of Distributed Cognition as a Theoretical Framework ........................................... 25

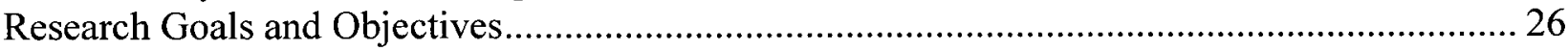

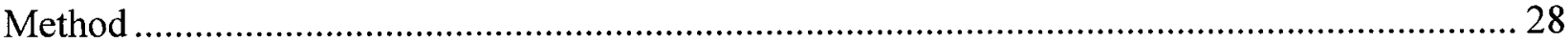

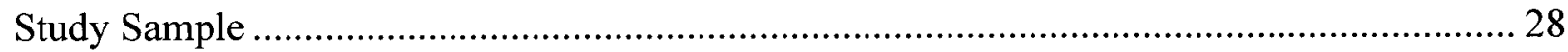

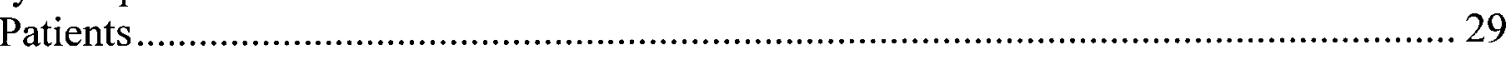

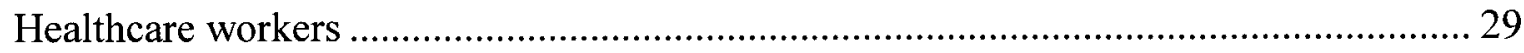

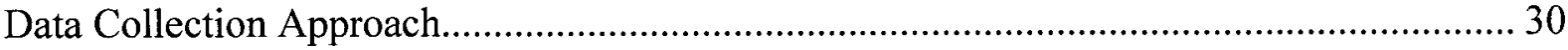

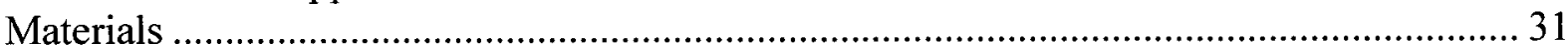

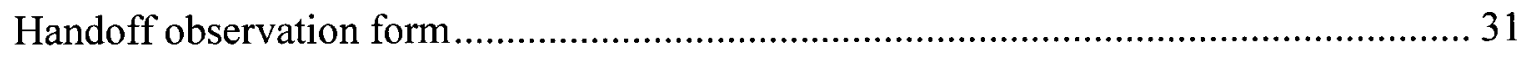

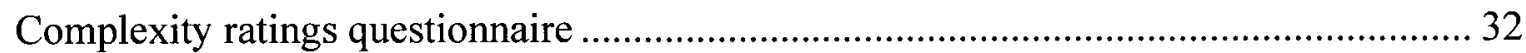

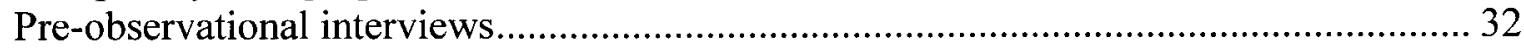

SBAR (Situation Background Assessment Recommendation) Protocol........................... 32

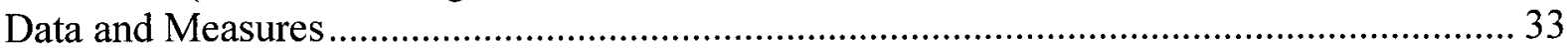

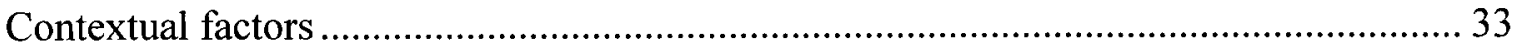

Information flow/dissemination of information............................................................ 33

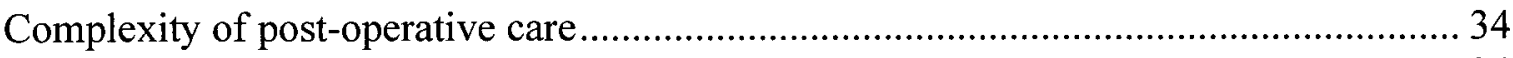

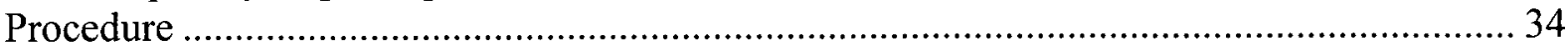


Participant consent and recruitment............................................................................... 34

Pre-observational interview ...................................................................................... 36

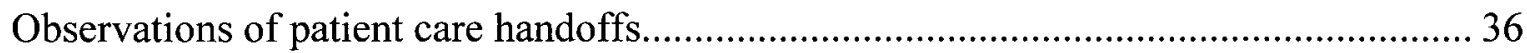

Administration of the complexity ratings questionnaire.................................................. 37

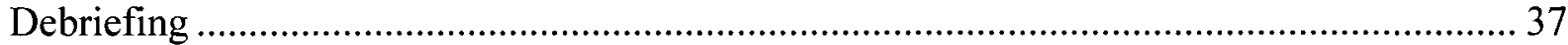

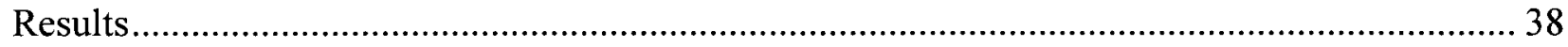

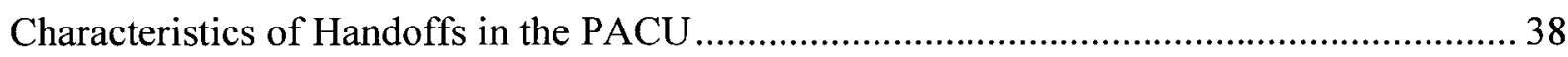

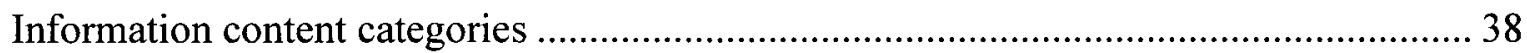

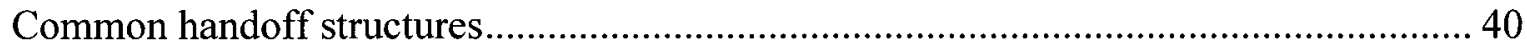

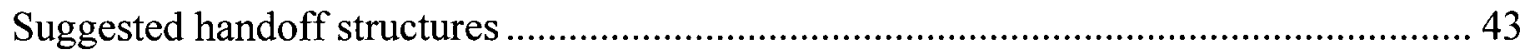

Gaps between suggested and actual information structures ........................................... 44

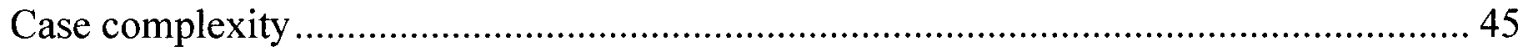

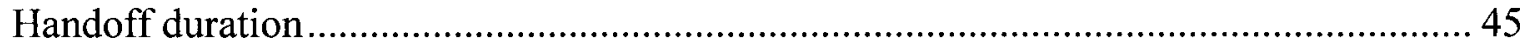

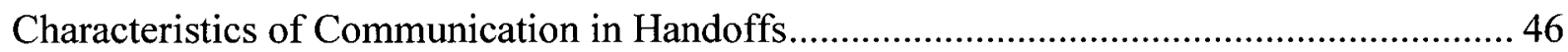

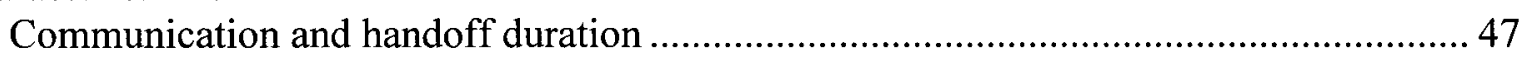

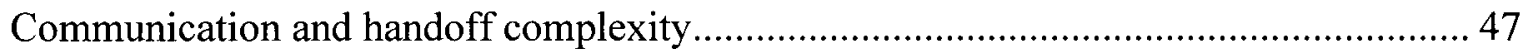

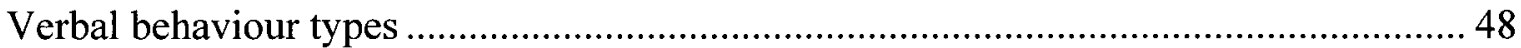

Relationships Between Suggested Structure and Variables of Interest ................................ 52

Verbal Behaviour Types and Information Categories ...........................................................5 53

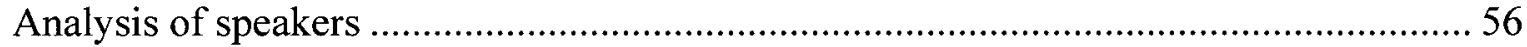

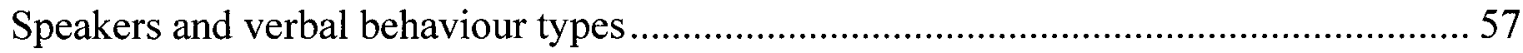

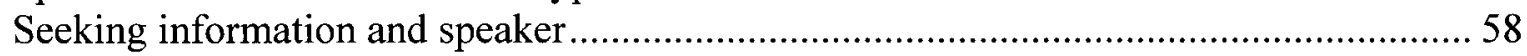

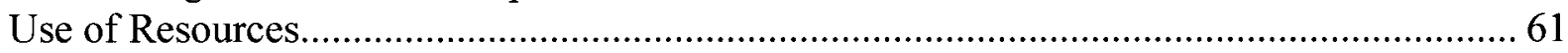

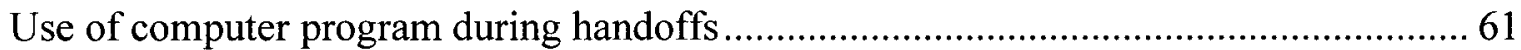

Verbal behaviour types with and without use of the computer program ........................ 63

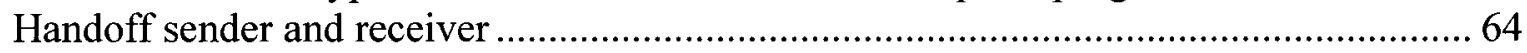

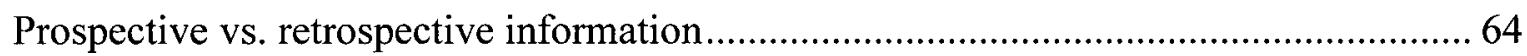

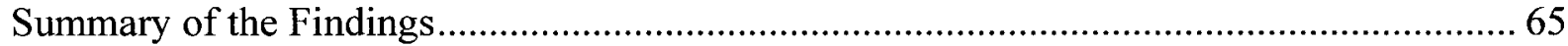

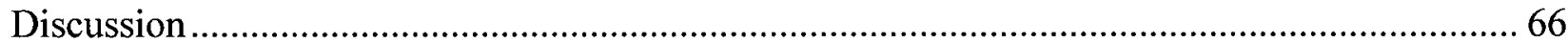

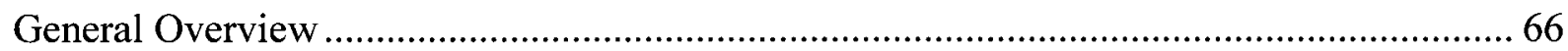

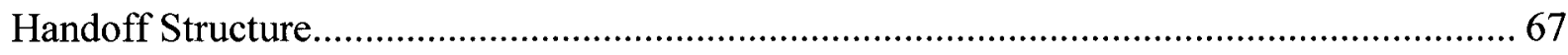

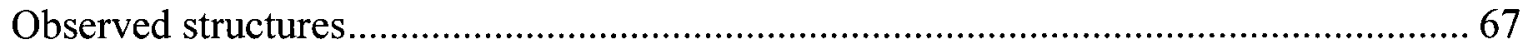

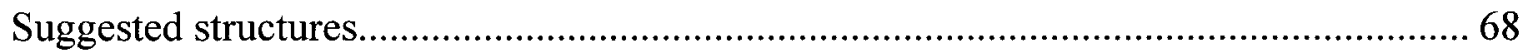

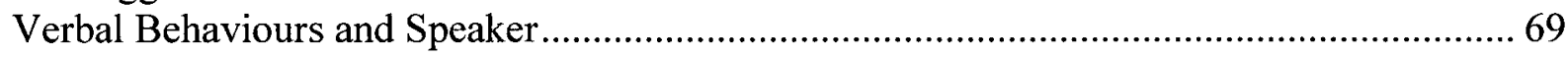

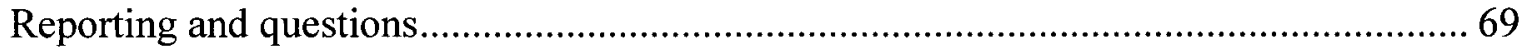

Handoffs Without and With Use of the Computer Program....................................................6 69

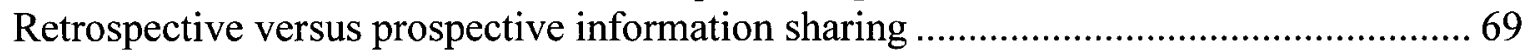

Information content and Periop Manager ..................................................................... 70

Focus on Retrospective Information Sharing ............................................................... 71

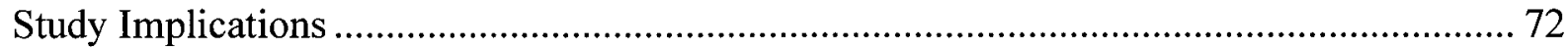

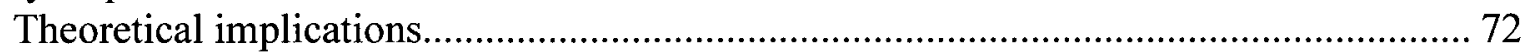

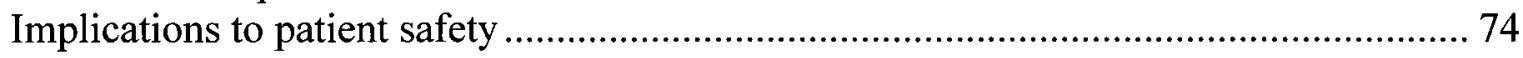

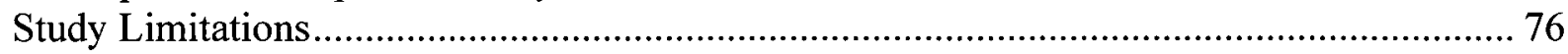

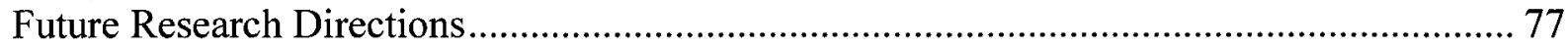

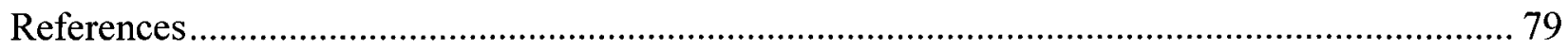

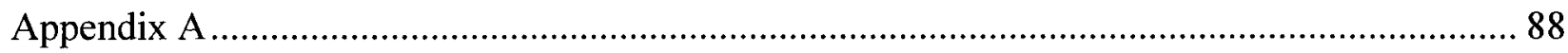


Appendix B

Appendix C .

Appendix D.

105

Appendix E

Appendix F. 


\section{List of Tables}

Table 1: Information Content Categories, Descriptions, and Examples..................39

Table 2: Examples of Content Categories.......................................... 41

Table 3: Suggested Content Structures According to Pre-observational Interviews.......43

Table 4: Verbal Behaviour Categories, Descriptions, and Examples...................49 


\section{List of Figures}

Figure 1: Frequencies of observed discussions involving specific content categories.

Figure 2: Flow chart detailing the common informational structure that emerged from handoff observations. The figure also displays content category names (general category, and sub-categories) and the breakdown of information content reported by anesthetists. The three content levels in the flow chart details the hierarchy of information.....

Figure 3: Relationship between the number of utterances per handoff and the handoff duration.

Figure 4: Relationship between the number of utterances per handoff and the complexity of each handoff

Figure 5: Frequencies of high-level verbal behaviour categories as a function of the proportion of total utterances.

Figure 6: Frequencies of verbal behaviour type sub-categories relative to the total number of utterances. 52

Figure 7: Frequencies of verbal behaviour types as a function of the information category and relative to the total utterances for each verbal behaviour type .55

Figure 8: Frequencies of questions, replies, and reports as a function of the information category and relative to the total number of utterances for each verbal behaviour type over the four information categories...... .56

Figure 9: The proportion of total utterances relative to healthcare profession. .57

Figure 10: Speaker-specific verbal behaviours relative to the total number of utterances 
Figure 11: The frequency of questions and replies by speaker for the information category of patient status relative to the total number of utterances for that verbal

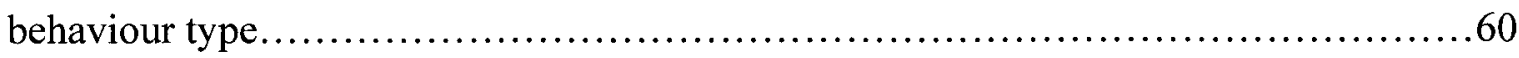

Figure 12: The frequency of questions and replies by speaker for the information category of future care plan relative to the total number of utterances for that verbal

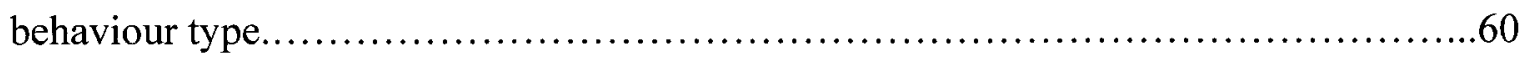
Figure 13: The frequency of verbal behaviours for handoffs without and with the use of a computer program relative to the total number of utterances for each type of verbal behaviour.

Figure 14: Proportion of planning and briefing relative to the total number of utterances

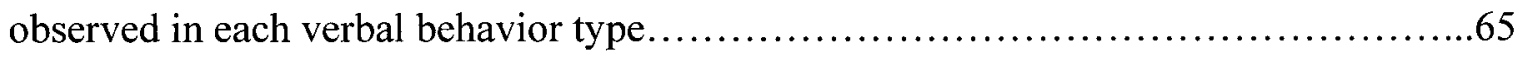




\section{List of Appendices}

Appendix A: Patient Information Sheet and Consent..........................88

Appendix B: Handoff Observation Tool....................................91

Appendix C: Complexity Ratings..........................................93

Appendix D: Questions for the Semi-Structured Interview....................105

Appendix E: Supplementary Data Tables..................................106

Appendix F: SBAR "Background" Report to a Physician About A Critical Situation 


\section{Communication in Patient Care Handoffs}

And Implications to Safety

In today's world of advancing medical technologies, the demand for the multitude of treatments and services available has drastically increased along with the number of patients who require these interventions (Van Eaton, Horvath, Lober, \& Pellegrini, 2004). Advancements in medicine have also been followed with increased concern for patient safety. Growing evidence has shed light on the phenomenon of preventable adverse patient events in healthcare, including serious harm or death. In the United States, medical errors are estimated to be the eighth leading cause of death, a higher instance than that of motor vehicle accidents, breast cancer, and AIDS combined (Varkey, Karlapudi, Rose, \& Swensen, 2009). Globally, one in ten patients in developed countries are harmed while receiving hospital care (WHO, 2008), a staggering number for a service that strives to provide care. Currently, there are several processes in healthcare systems that do not have standardizations in place and this has been documented as a source of vulnerability to error. (Leonard, Graham, \& Bonacum, 2004; O'Byrne, Weavind, \& Selby, 2008; Pesanka et al., 2009; Schultz, Carayon, Schoofs Hundt, \& Springman, 2007). Of particular interest is the process of patient care handoffs. The transfer of a patient's care from one healthcare worker to another or from one area of care to another involves high-pressure communication contexts that are often vulnerable to information loss and can sometimes lead to medical error.

It has become apparent to many health organizations, through evidence-based practice, that initiatives must be taken to reduce and eliminate adverse medical events. Many research studies have made the impartment of knowledge concerning sentinel 
events possible and patient safety initiatives have become a top priority for the majority of health organizations as a result (including The Canadian Patient Safety Institute, The National Patient Safety Foundation, and The World Health Organization) (Baker, Day, \& Salas, 2006). The foundation for the development of standards of care, training programs, and procedures for the minimization of medical errors has been laid. Both safety and quality of patient care are now at the forefront of medical research as hospitals and health organizations move forward in an attempt to adapt to the current challenges and obstacles they face when it comes to providing effective and safe patient care.

The purpose of the present thesis was to capture current practices and procedures for handoffs in the hospital setting. Specifically, the objective was to identify the flow of information transfer and communication between staff and information technology by mapping out the processes involved in transferring a patient from the operating room (OR) to the recovery room (often referred to as the post-anesthetic care unit or PACU). A secondary objective of this research was to provide preliminary suggestions concerning standardizations in patient handoffs. In the long-term, the results of this thesis can be used to aid in the development of tools and devices to facilitate successful patient transitions and thereby, potentially minimize the risk of adverse medical events in the future.

\section{An Overview on Patient Safety}

Patient safety is an overarching component of patient care and is defined by The World Health Organization (WHO) as "a newer discipline in healthcare that emphasizes the reporting, analysis, and prevention of medical errors which often lead to adverse healthcare events" (WHO, 2008). Patient safety incorporates several healthcare topics 
such as: communication and patient care handoffs, sentinel event reporting, hand hygiene, atmospheres that promote education and learning, universal protocol, and the culture of patient safety (Varkey et al., 2009). Despite reports that failures in safety are responsible for up to 17 billion dollars in losses nationally (U.S), there are still some hospital administrations and affiliates that remain hesitant to fully support the patient safety movement (O'Byrne et al., 2008). Such lack of support fails to recognize that sentinel events are responsible for longer hospital stays, lawsuits, higher costs of care, and insurance payouts; these events combined create much greater expenses for the healthcare system (O'Byrne et. al., 2008). Patient safety is a discipline that requires extensive research and empirical evidence before standardizations and tools for improving patient care safety can be implemented. Fortunately, medical practice currently exists in an era that emphasizes and promotes a culture of safety. As steps are taken towards developing safety competencies and expanding the current empirical knowledge bank on medical error, hospitals, medical staff, and patients alike are slowly moving towards a new culture of patient safety. Once fully developed, this culture will be supportive of patient safety initiatives, procedures, and protocols.

Since the release of the report "To Err is Human" (Kohn, Corrigan, \& Donaldson, 1999), researchers have been collecting a multitude of data on the instance of medical error, especially in relation to the operative process (Baker et al., 2006; WHO, 2008; Windle, Mamaril, \& Krenzischek, 2007). Errors committed during the operative process, whether medical, administrative, or systems related, account for almost half of the adverse events that result in death or disability and yet every year more than 100 million people require surgery (WHO, 2008). Surgical interventions have become more advanced 
and complex and as seen above, can involve serious health risks. These factors have prompted empirical researchers to study the implications for quality and safety of patient care in these stages of inpatient hospitalization. Although there has been plenty of data collected on the facilitators and barriers to effective patient care in the OR itself, little empirical attention has been drawn to the safety practices of care throughout the entire perioperative process (Windle et al., 2007). This process not only includes the operative procedure, but also the transfer of a patient to the recovery room, the surgical postoperative unit, and their discharge.

\section{The Importance of Patient Safety in Critical Care Environments}

The present thesis was focused on the critical care stage of recovery that occurs after surgery. This stage is sometimes referred to as part of the perianesthesia period (e.g., care that begins in the OR and ends in the post-operative surgical unit). The recovery room is responsible for the care of patients during one of the crucial points in their hospitalization and its environment is both unique and high-risk. Anesthesia and surgery are often risky on their own, and along with the high level of activity in the recovery room (including multiple patient handoffs and constant activity) there is an increased susceptibility to error (Windle et al., 2007). Although patient safety is a top priority in most healthcare settings, several researchers have noted that in the current literature, little is known about the culture of patient safety, including the reporting, identification, and active avoidance of adverse medical events in the perianesthetic stages (Kaafarani et al., 2009; Ross \& Ranum, 2009). Promoting a culture of patient safety, especially in critical care settings, is essential to providing safe healthcare, especially because these hospital units incur challenges to safe practices due to the complex nature of their patients (Windle et al., 
2007). In a study by Kaafarani et al., (2009) it was demonstrated that the post-operative recovery room and the operating rooms in 30 American hospitals had slightly worse safety cultures than other clinical work areas in the same hospitals. This is why it is essential to continue researching patient safety in specific environments, such as the recovery room, to raise awareness and involvement in the promotion of safe and quality healthcare across the board.

As medicine evolves, researchers and medical experts have come to appreciate the need to understand the underlying processes that can lead to adverse medical events (Coiera, 2006). In turn, several patient safety research endeavors have shifted towards obtaining first-hand knowledge in various hospital settings. Traditionally, patient safety research has used retrospective data on sentinel events to draw conclusions about the events leading to medical error, but has been criticized for being incomplete (Christian et al., 2006). These research studies can sometimes lack details, such as communication processes, concerning the context of the adverse event.

Another approach to patient safety research investigates the naturalistic context of the processes and procedures, such as patient care handoffs, which can lead to potential errors. Inefficient communication, especially during critical points of care such as the handoff, is more susceptible to medical error. For example, in a study of closed malpractice claims by the Controlled Risk Insurance Company/Risk Management Foundation Staff (CRICO/RMF), it was found that $24 \%$ of missed diagnoses were a result of inadequate transfers of patient care (Kachalia et al., 2007). Research into these processes and environments is therefore critical for the benefit of quality and safety of healthcare in the future. 


\section{Handoffs}

For the purposes of this thesis, a patient handoff, also referred to as a patient care transition or hand over, is defined as the transfer of relevant information and knowledge concerning a patient's medical status (including their current condition, anticipated changes, history, and future care plan) from one healthcare worker or team of healthcare workers to another. The primary objective of the care transfer is to relay accurate information that is provided and received in a timely, clear, and complete manner (JCAHO, 2008). Care transfers are a multidisciplinary effort and require effective and efficient communication in order to maintain quality, safety, and continuity of care; all of which are essential components of healthcare.

According to O'Byrne et al. (2008), the patient handoff is one of the most important points in the healthcare process for the exchange of critical information. There are three major changes that occur in the process of a handoff and these include a change in the provider, location, and/or unit or department of care (Horwitz, Meredith, Schuur, Raghavendra, \& Grace, 2009). In hospitals today, the moment a handoff to a new health care provider and unit occurs there is an associated risk of the transfer being made into an environment that interacts, flows, and follows procedures differently (Pesanka et al., 2008; Schultz et al., 2007). These variations in care provision across healthcare settings are commonly a result of a lack of handoff standards or guidelines in place. Therefore, although handoffs are a necessary component of patient care, they can increase susceptibility to medical error through poor transfers of information, authority, and responsibility for patient care (Schultz et al., 2007). 
In most situations when a handoff is critical to the success of a task, standard processes and procedures are practiced regularly. In aviation for example, the transfer of an aircraft from one air traffic controller to another is practiced routinely and the moment-by-moment communication skills involved are observed by experts in order to improve efficiency and avoid potential error (Solet, Norvell, Rutan, \& Frankel, 2005). In healthcare at this point in time, organizations can only go so far as to say they have made "recommendations" for improving handoffs and avoiding potential error. It is important that healthcare organizations learn from other empirically supported handoff methods employed in other industries (e.g., crew resource management in aviation; Helmreich, 2000).

\section{Patient Safety and Communication}

One aspect of the recent patient safety concerns and initiatives that warrants special attention is the role of communication. Effective communication is arguably the most important tool in healthcare as it is required for almost every position and activity in the hospital (O’Byrne et al., 2008; Parker \& Coiera, 2000). Patient care involves the coordinated efforts of multiple individuals and professions who need to share information and plan patient care accordingly (Baker et al., 2006; Yeager, 2005). Although there has been a recent push towards the integration of various technologies as interventions for communication issues, more attention is still required towards understanding the underlying basic human-to-human information exchanges and methods of communication processes in hospitals (Coiera, 2006; Parush et al., 2009; Parush et al., 2010). 
It is important to first investigate the components and concepts of current human communication events before these interventions are introduced. The whole systems of communication in healthcare today include people, organizational structures, and technology and they are the formal or informal organizational structures that support the delivery, channeling, and interactions for communication exchanges (Coiera, 2006). According to various research studies (Alvarez \& Coiera, 2006; Safran, Sands, \& Rind, 1999; Tang et al., 1996) including one specific study (Alvarez \& Coiera, 2006) that found verbal communication between staff members to be responsible for $90 \%$ of all information exchanges, communication is the largest information network and also comprises the largest "space" of information in medicine. Unfortunately, this "space" is highly vulnerable to errors (Coiera, 2006; Parker \& Coiera, 2000).

\section{Communication breakdowns}

Constructive information flow is a major component of a successful healthcare team and at the root of this is the importance of efficiency and effectiveness of communication. Unfortunately, poor communication appears to be commonplace in North American hospitals where it was listed as the major cause of sentinel events across medical disciplines from 1995-2006 (O'Byrne et al., 2008). In 1999, it was disclosed that between 44,000 and 98,000 deaths occur annually as a result of preventable error (Kohn, Corrigan, \& Donaldson, 2000). Furthermore, communication errors are the most frequently documented cause of sentinel events, as evaluated by the Joint Commission on Accreditation of Healthcare (JCAHO; Odom-Forren, 2007). Among the many patient safety goals set out by the JCAHO is the goal to "improve the effectiveness of communication among health care providers" (JCAHO, 2008). In a study of 16,000 in- 
hospital deaths, when it came to the comparison of adverse outcomes as a result of inadequate skills versus communication, failures in communication were more than twice as likely to cause harm (Dykes et. al., 2007; Wilson et al., 1995). Ineffective communication was also reported to have a negative impact on care provider satisfaction and delivery (Ayse \& Xiao, 2006). These adverse healthcare events are preventable and despite popular belief that the integration of technological devices and tools will correct the problem, it is also important to address these faults on a theoretical level (Ayse \& Xiao, 2006).

Until recently, research has focused on disease and procedure complexity as some of the main sources of medical error, however, it is now known that health care providers themselves and the systems they work in (whether in a team or individually) are a major contributing factor to the occurrence of adverse health events. The efficiency of communication in hospitals is a known problem and understanding the nature of these breakdowns is essential for a solution in the future. In a study involving observations of 421 communication events between healthcare providers, almost one third of those events were classified as breakdowns (Lingard, Espin, \& White, 2004). Research has suggested an emerging pattern of the high prevalence of communication breakdowns between physicians, nurses, patients, and other members of the healthcare team (Hardeep, Aanand, Raghuram, \& Peterson, 2007). Flawed communication resulting from unclear lines of authority across professions, disconnected reporting systems, and fragmented systems that lead to numerous handoffs are some examples of how system failures can lead to patient harm (Hardeep et al., 2007). For the purpose of the present study, the interactions between healthcare providers and their primary work environments are important for 
understanding the root cause of communication failures. Breakdowns in communication occur when information transferred from one healthcare provider or team of healthcare providers to another is misinterpreted, unclear, missing, or wrong altogether. Breakdowns can lead to delayed care, information loss, inaccurate diagnoses, and inconsistency in care, all of which can be responsible for sentinel events (Bell et al., 2009).

In summary, communication is a very important component to patient safety as it takes up the largest "space" of information in medicine. The structure of human communication events supports the channeling, delivery, and interactions for information exchange. However, these events are susceptible to breakdowns and poor communication has been cited as a major contributing factor to sentinel events. Due to the fact that healthcare organizations rely so heavily on communication for patient care, it is important that these processes be investigated. Effective communication is considered to be critical during the transfer of patient information from one provider to another. In these situations, miscommunications or missed information can result in serious patient harm and even death (JCAHO, 2008). The following section addresses communication and information flow in the patient care handoff.

The Importance of Communication During the Patient Care Handoff

Effective and accurate information flow between healthcare providers during patient care handoffs is crucial and the consequences of error become significant for both the patient and the healthcare worker. In a study of the processes of post-operative care, it was discovered that the patient outcome was directly related to communication and care coordination (Odom-Forren, 2007). Additionally, in a study that documented 2,455 
sentinel events, $75 \%$ of patients who died as a result of their care process died because of communication errors (Leonard, Graham, \& Bonacum, 2004).

In a situation where the handoff results in ineffective information transfer, confusion arises along with concerns for the safety of the patient. The search for missing information or clarification of treatment orders can lead to delays in care and/or adverse events (Foster-Hunt, 2009; Schultz et al., 2007). The negative consequences of such gaps in communication are felt by all parties, as the workload and stress for the healthcare providers increases along with risks to the patient. Poor communication concerning patient-relevant details during a transition can also lead to inconsistencies in care, which has been identified as a threatening factor to patient safety (Greenberg et al., 2007).

\section{Consequences of ineffective handoffs}

The following scenario details a negative patient experience as a result of discontinuity of care evolving from ineffective handoffs during a perioperative stay in a hospital. The case was analyzed and discussed at a patient safety conference in 2006 (WHO, 2008). The scenario details the events surrounding a multidisciplinary healthcare team who ran into several communication failures in the care of a specific patient and resulted in a malpractice claim. There were errors made at multiple points during the patient's stay at the hospital. It is important to note how critical the handoff process was in this situation for conveying relevant and vital information, and how there were several cross-professional communication barriers. Despite several attempts to communicate complications, a lack of effective communication and standardization in the handoff process led to serious patient harm. 
Twenty-year old Patrick Dwight suffered from multiple birth defects and mild Diabetes Insipidus, a condition in which the kidneys produce an abnormal amount of diluted urine. Patrick depended on his family for all aspects of his life. He couldn't talk, but communicated with facial expressions and the use of a computer translator. He was admitted to the hospital for a right hip replacement with a surgeon who had previously done his left hip. Patrick's mother reminded the surgeon of her son's high sodium levels the previous time which resulted in an extended stay at the hospital. The surgeon told Patrick's mother to tell the anesthesiologist that day and she also made sure the nurse practitioner wrote this information on the pre-operative chart. During the surgery, the resident was called to another case and an orthopedic fellow completed the surgery. That day the attending surgeon went away on vacation. The fellow discharged Patrick from the OR but, unaware of his condition, he did not order serial labs or monitoring of fluid intake. Once on the floor, Patrick's mother told a second nurse about his condition but she did not follow up on it assuming that the physician had included this in his orders for the patient. After 4 days, Patrick began experiencing seizure-like activity, slipped into a coma, and developed pneumonia. After the problem was corrected, an MRI showed significant brain damage. Patrick was discharged into a long-term care facility and can no longer communicate in any way. A malpractice suit was settled for more than one million dollars (CRICO/RMF, 2007).

Hospital personnel are often challenged with complex patient scenarios, such as Patrick's case detailed above. It is apparent that communication can become a barrier to safety in such circumstances. The above scenario highlights how multidisciplinary communication failures can contribute to adverse patient events, as there are several 
instances where staff members did not relay patient information to the incoming care providers. The following section investigates the literature on the social context of communication errors, especially with regards to collaboration between care providers in healthcare.

\section{Multidisciplinary Communication and Care}

Multidisciplinary communication, in the context of medicine, involves non-verbal and verbal interactions between various healthcare disciplines (e.g., from emergency room doctor to surgeon, nurse to doctor, anesthetist to nurse, nurse to porter). All interactions that are shared with each other regarding the patient(s), whether it be to relay drug information, participate in patient rounds, treatments, or shift reports are included in this dynamic form of communication. Multidisciplinary collaboration, also referred to as multidisciplinary teamwork, involves healthcare staff coming to a mutual agreement for the purpose of achieving mutual goals (Dechairo-Marino, 2001; Kramer, Parush, Brandigampola, \& Momtahan, 2010) and involves a combination of skills and attitudes (Baker et al., 2006; Yeager, 2005). These interactions are critical for the coordination of patient care because each discipline brings a slightly different picture of the patient care plan to the table. As such, successful collaboration or teamwork should occur in an environment of mutual understanding and respect, where each individual is free to divulge their concerns. People who work well in teams believe that their work cannot be carried out effectively without the other members' input (Norsen, Opladen, \& Quinn, 1995). In turn, the successful integration of the team's assets and medical knowledge should facilitate increasingly synchronized care. 
Although healthcare is delivered by a multitude of care providers of different specialties and backgrounds, patient safety and quality of care has historically been practiced as an individual endeavor rather than a team effort (Xyrichis \& Lowton, 2008). The empirically supported benefits of multidisciplinary communication and teamwork include: increased job satisfaction, less delays in care, increased efficiency, increased patient satisfaction, and fewer medical errors (Yeager, 2005). These benefits are often experienced when healthcare processes are standardized and yet standardization of healthcare processes like patient handoffs has still not been widely implemented.

Multidisciplinary handoffs are most commonly the rule rather than the exception when it comes to care in inpatient settings. When communication in these dynamic teams is facilitated, the environment becomes supportive of collaborative and effective care judgments and decision-making between health care workers. There are, however, several factors working against rather than for effective communication in a multiprofessional healthcare team.

\section{Multiple care providers}

In situations where different healthcare providers are involved, there are also different individual information requirements and this creates complexities in the communication of relevant patient information (The Royal College of Surgeons, 2007). Inevitably there will be different ideas of what is relevant to a case and this is why it is a challenge to develop and implement a standardized approach to handoffs.

Not only are the care providers themselves a factor, but also the contexts of care. In some cases, patients are transferred to beds in inappropriate specialty areas where the multidisciplinary dyad must adapt to communicate about information and knowledge that 
they do not share. Patients can also be moved around to different beds depending on availability, the need for a bed for a critical patient, or scheduled bed bookings; also creating communication barriers. In a study by Williams et al., in 2007, there were 38 reports where moving a patient to a different bed resulted in decreased patient familiarity and the need to constantly update new staff on medical details. A loss of patient familiarity is a potential facilitator of information loss or miscommunication, especially when it results in a need to recollect patient information. In effect, every instance of information exchange holds the potential for miscommunication.

\section{Cross-professional communication}

Horwitz et al., (2009) argued that interpersonal communication failures across professions were prominent in patient care handoffs and one of the reasons cited were the boundaries created by different "social worlds". In the past, and even today, people are socialized in learning environments, as well as in the workforce, with similar people. In a hospital setting, this translates to physicians socializing with physicians and nurses with nurses. When it comes time for these various professions to join together in a team for a patient's care, there can be communication problems because of these different socializations. This diverse team has not always had the time to appreciate one another or to develop patterns of communication and compounded with the lack of content standardization in the handoff process, there is the potential for a disorganized interaction.

\section{Hierarchical working relationships}

In an ethnographic study of the dynamics among multidisciplinary interactions, nurses reported that they felt undervalued, weren't directly involved in decision-making, 
were interrupted, and were reminded of the "power distance" between themselves and the physicians (Alvarez \& Coiera, 2006). The power distance refers to a situation where a subordinate staff member may not want to speak up about their concerns or question the authority of their superior (O’Byrne et al., 2008; Leonard et al., 2004; Tan \& Macario, 2008; Williams et al., 2007). In medicine, a by-product of this power distance is a limited extent of communication between nurses and physicians directly. In a study of 46 patients in an ICU, there were a total of 178 activities per patient, per day and out of the total activities observed, only $291(9 \%)$ were verbal communications. Only $2 \%$ of the observed activities involved verbal communications between nurses and doctors. Conversely, $37 \%$ of the error reports recorded involved nurse-physician communication (Donchin et al., 1995). As evidenced by this study, multidisciplinary communication barriers are a concern.

Other industries, such as aviation, have run into similar communication problems and have recognized that creating a culture where people feel secure expressing their concerns, is a key factor to safety. American Airlines developed a model of communication called CUS to combat the issue. The acronym stands for "I'm concerned, I'm uncomfortable, this is unsafe or I'm scared" and has been shown to be very effective in facilitating efficient and effective communication (Leonard et al., 2004). Adapting other models of communication, such as CUS, that have been proven effective in industry, is an important concept for healthcare. Clinicians need to be able to be direct with their questions and concerns and in order for this to happen, an explicit safety culture has to be present (Leonard et al., 2004). 


\section{Summary}

In summary, effective multidisciplinary communication is highly critical to the successful co-ordination of patient care in a healthcare environment. The boundaries created by power distances among professions, differing social groups, and multiple care providers are some of the factors that can contribute to communication breakdowns. These breakdowns can result in delayed care, discontinuity of care, and in some cases, medical error. The aforementioned barriers to effective multi-professional teamwork are issues that hospitalists and healthcare institutions are currently working on improving. The promotion of the culture of patient safety, through the reporting, discussion, and analysis of medical error and vulnerabilities to error is a means to this end goal. Healthcare professionals need to be educated on areas of vulnerability in their practice and hopefully this will encourage clinicians to work together as a team, to combat these issues.

In order to develop communication solutions that work in healthcare, several research studies have investigated the communication modes employed in healthcare settings and evaluated these in terms of their facilitation of performance during patient care. Depending on the environmental circumstances, different communication approaches can provide different benefits or consequences to effective healthcare. The following section describes current methods and modes of communication.

\section{Current Methods and Modes of Communication in Healthcare}

The methods and modes of communication in a hospital setting can vary depending on communication and organizational factors such as the available communication infrastructure, the organizational structure, and the specific clinical environment (e.g. 
ICU, PACU, ER; Coiera \& Parker, 2000). Communication, which involves interactions between multi-professional patient care teams, can take on many different forms including paging, hallway exchanges, verbal or written reports, audio recording, notes, messages, or phone calls and are all collectively referred to as channels of communication (Coiera, 2006).

There are two different modes of communication that have been observed in the hospital setting in the process of patient care transitions, synchronous and asynchronous communication. Synchronous communication occurs when a handoff is carried out in real-time, most commonly completed in a face-to-face manner (Alvarez \& Coiera, 2006; Greenberg et al., 2007). In other situations, it can be done over the telephone, usually if the sending staff member is not available to transport the patient. Asynchronous communication, on the other hand, is not completed in real-time. This communication mode commonly employs the use of technology or tools and therefore, usually takes on the form of patient notes or charts, checklists, audio recording, whiteboards, computer devices, personal digital assistants, or voicemails. At the study hospital, patients are transferred from the OR to the recovery room synchronously by means of a face-to-face interaction and information is exchanged upon immediate receipt of the patient. Therefore, the review to follow will focus solely on synchronous communication.

\section{Synchronous communication}

In a study by Coiera and Tombs (1998), synchronous communication was identified as the most preferred means of communication throughout the experimented hospital and this finding has been confirmed by multiple research studies since then (Coiera \& Parker, 2000; Coiera, 2006; Dykes et. al., 2007). There were several reasons listed and among 
them was the desire for the social facilitation of the face-to-face interactions, the ability to clarify and ask questions with immediate responses, and the ability to confirm the transfer of responsibility for the patient (Coiera, 2006). Asynchronous modes of communication, e.g. audio recording, are not always equipped to facilitate these types of information seeking interactions (Kerr, 2002; Webster, 1999). Furthermore, the inability to ask questions and clarify information goes against the JCAHO's patient safety recommendations for handoffs (JCAHO, 2008). This could be why in a recent study of communication events in a North American hospital it was documented that only $9 \%$ of handoffs were performed asynchronously by means of an audiotape (Alverado et al., 2006).

In a recent study of patient handoffs during nursing shift reports, researchers found that face-to-face communications were the most frequent means of information exchange. These communication events were highly interactive and it was documented that additional requests for information increased as a function of the level of organization of the handoff (disorganized to highly organized; Foster-Hunt, 2009). Based on empirical evidence, the largest source of information transfers is conversations between individuals (Coiera, 2006; Foster-Hunt, 2009; Parker \& Coiera, 2000) and several studies have attempted to quantify these communication events. In a study by Tang et al. (1996) about $60 \%$ of clinicians' time at work on the floor was spent in conversations; Safran et al. found very similar results in their study where $50 \%$ of information exchanges were faceto-face (Safran, Sands, \& Rind, 1999). Patterson et al. (2004) have suggested, through observation and analysis, that if verbal exchanges can maintain an interactive nature, they could be a potential strategy for combating information loss. This idea was supported by 
the shift report research mentioned above (Foster-Hunt, 2009). Furthermore, during simultaneous interactions, health care providers are given an opportunity to discuss or question a fellow colleague's point of view and this can sometimes lead to new ideas or patient care solutions (Zwarenstein et al., 2007).

Synchronous communications have also been known to incorporate the use of tools for information exchange including patient charts, written reports and notes, computers, and paper forms in addition to the verbal interaction (Foster-Hunt, 2009). In a study by Alverado et al. (2006), 65\% of observed information exchanges involved the use of similar tools in combination with conversations. In a recent observational shift handoff study, tools were referenced an average of 16 times per handoff and longer verbal handoffs were related to an increase in the use of tools (Simoneau, 2009). It is apparent that tools are a necessary component to patient handoffs. Despite the various benefits of synchronous communication, there are skeptical criticisms of simultaneous exchanges of patient information as they are often subject to the strains of complex medical environments.

\section{Communication problems associated with synchronous communication}

The shortcoming of synchronous communication is its interruptive nature. When conversing with an individual, it is easy for diversions from the topic because there is an opportunity given for questions to be asked, other medical interruptions to occur, and/or external distractions. Interruptions in the handoff process are what create the problem for the flow of communication and can lead to medical error. Even if the interruption takes only seconds away from the action, it can result in memory failure for the clinician (Moss, Xiao, \& Zubaidah, 2002). In interruption-driven environments, the cognitive 
resources of healthcare facilitators are taxed as they work to perceive and communicate information (Patel, Zhang, Yoskowitz, Green, \& Sayan, 2008).

Simultaneous communication methods can also be inefficient for clinicians in terms of availability. Co-workers may not be available for face-to-face conversations; they may be busy or unreachable by telephone and as a result, tasks may remain incomplete due to a lack of necessary information. In a study by Coiera and Tombs (1998), of 56 observed call attempts, one third of telephone call attempts failed due to a busy line. In this situation, the care facilitator has to take time away from patients to collect the relevant information and until contact is made with the other healthcare provider, patient care is delayed (Coiera \& Parker, 2000).

Finally, simultaneous verbal handoffs have been criticized for being oriented towards retrospective patient information. Research has demonstrated a tendency for healthcare staff to focus on sharing past information (e.g. what medications were administered in the OR) versus information sharing that is prospective (e.g. what medications to give over the recovery period; McMahon, 1990). This is a concern because empirical evidence points to the benefits of prospective information sharing for improved recall and retention of information along with the facilitation of care plans (Kihlgren, Lindsten, Norberg, \& Karlsson, 1992; \& Luikkonen, 1993; McMahon 1990).

\section{Summary of synchronous communication}

Synchronous communication in healthcare has its strengths and weaknesses. This mode offers social facilitation and the opportunity to convey and/or interpret facial and verbal cues during patient care handoffs. However, face-to- face simultaneous communications have been known to be susceptible to interruptions, especially in such 
dynamic environments as healthcare. The use of tools (patient charts, computers programs, etc.) is common during patient care handoffs and contributes to the dissemination of information during these synchronous interactions.

Summary of Handoffs

In summary, efficient and effective handoffs involve interactive communication and tools that facilitate the retention of pertinent patient information while minimizing the potential for information loss. During patient care handoffs, these communication efforts involve multidisciplinary communication where team members feel secure expressing their concerns and ideas for the patient's care plan. Accurate information transfer is a product of the cultural, social, and technological environments surrounding the cognitive tasks involved in a handoff. Due to the fact that previous research has demonstrated how all of these factors are critical to the success of patient care handoffs (Coiera et al., 2000, 2006; O’Byrne et al., 2008; Pesanka et al., 2008), the present study will structure observations around a theoretical framework that emphasizes the capture and analyses of these contextual factors.

\section{Distributed Cognition as a Theoretical Framework}

Distributed cognition is a useful framework for understanding, defining, and categorizing contextual and social factors that can lead to medical error. In the dynamic environments of healthcare, work is distributed across professions, tools, and work areas. There is a complex cognitive system underlying communication processes that direct memory, action, perception, and decision-making (Patel et al., 2008). Distributed cognition is a theoretical development that attempts to put cognitive concepts into practice in an effort to study dynamic systems in a manner that can facilitate the future 
improvement of task performance. This discipline is interested in studying how cognitive activity is distributed across the mind of the individual, groups, and external artifacts (Hollan, Hutchins, \& Kirsh, 2000). It is founded in a belief that the research focus needs to be shifted away from mentalistic assumptions about the individual and more towards a focus on the cognitive system as a whole, including the team of people required for the task and the artifacts they employ (Artman \& Garbins, 1998; Hazelhurst, McMullen, \& Gorman, 2007; Hollan et al., 2000; Rogers \& Ellis, 1994).

The components of the distributed cognitive system include both internal and external representations. Internal representations are the structure and collection of knowledge in the mind of the individual, whereas external representations are the structure and collection of knowledge in the external environment (e.g. groups of people, artifacts). Information and cognitive behaviours can be distributed within an individual mind, between individual minds, and between minds and artifacts such as tools (e.g. patient charts or computers), objects (e.g. the stethoscope), or symbols (e.g. writing, medical acronyms) (Hollan et al., 2000; Patel et al., 2008). Therefore, the unit of analysis in this theory is the entire cognitive system within which human actions occur and is constructed as a result of the coordinated work of each unit (individual, team, and artifacts) in the system (Artman \& Garbis, 1998). Researchers and subject experts view clinical workplaces as cognitive systems where cognitions are used for providing patient care through a collaborative team process involving the use of artifacts (Artman \& Garbis, 1998; Malhotra, Jordan, Shortliffe, \& Patel, 2007). Respectively, the recovery room was an appropriate observational environment because of its dynamic team environment and the high frequency of handoffs. 


\section{Distributed Cognition and The Patient Care Handoff}

Perioperative units, such as the recovery room, have been documented as complex and dynamic working environments (Kaafarani et al., 2009; Shultz et al., 2007).

Distributed cognition, with its foundations in the study of human behaviour across multiple environmental aspects, is a useful framework for studying patient safety with respect to the system as a whole. The present research focused specifically on the handoff process in the post-operative recovery room. Nurses in this unit are assigned to specific patients and their care involves individual practice, multi-disciplinary communication, and interactions with medical artifacts (e.g. patient charts). Activity in these hospital environments is highly distributed. Through ethnographic observations, the researcher was able to detail the workflow of the recovery room as a function of the channels of communication, thus providing a glance into the context of communication processes in healthcare.

The integration of social and technological factors into the complex communication process is both necessary and characteristic of handoffs (Simoneau, 2009). During the handoff process, medical artifacts (patient charts, computers, monitors, etc.) are very important tools for the support of quality and safety of care. The tools used can range from monitors, whiteboards, computers, computer-generated forms that are attachments to the patient chart, and temporary personal use handwritten notes (Simoneau, 2009). Through an understanding of how information is distributed among the healthcare team members and their medical artifacts, researchers can draw a clearer picture of how specific tools contribute to the performance of a handoff (Blanford \& Furniss, 2006). 
Likewise, the information flow between multidisciplinary healthcare workers is a critical component of the patient care handoff and the collaboration of care providers is vital to the delivery of effective healthcare (Hazlehurst, McMullen, \& Gorman, 2007; Zwarenstein et al., 2007). Understanding the roles of healthcare staff and the sequences of events that they engage in to complete their tasks is an important requirement for understanding the process of patient handoffs. The distribution of cognitive tasks across individuals as well as across artifacts can define the mechanics of the system (Artman \& Garbins, 1998; Blanford \& Furniss, 2006; Hazelhurst, McMullen, \& Gorman, 2007). It is apparent that work is often distributed across multiple sources of knowledge in patient care handoffs. Distributed cognition forms a framework for investigating the phenomena of communication by analyzing all components of the context within which it occurs and then transforming these multiple pieces of knowledge into an understanding of the procedures of handoffs as a whole. Observational efforts examined the various relationships between the healthcare staff and their available resources to draw conclusions about the acquisition and dissemination of knowledge and information in the hospital setting.

\section{Summary of Distributed Cognition as a Theoretical Framework}

Communicative processes are defined by the mechanisms involved in completing the task and as such, there are three distinguishing processes of distributed cognition. These cognitive processes are (1) distributed among members of the social group, (2) involve interactions between internal (mind) and external artifacts, and (3) are distributed across time over the entire structure of the environment (individual, groups, artifacts; Hollan, Hutchins, \& Kirsh, 2000). Other theoretical frameworks and methodologies such as 
situation awareness were taken into account when determining the appropriateness of distributed cognition for the present thesis; however, the theory of situation awareness does not place a focus on the use of artifacts.

Ultimately, insight into information exchange through the framework of distributed cognition may provide answers as to how and where barriers to communication in the handoff process can open the gap for information loss. As cited by Coiera and Parker (2000), in order for change to occur for the benefit of patient safety, researchers must fully understand communication phenomena within the natural environment. In dynamic environments such as healthcare, the theory of distributed cognition emerges as a relevant framework because the people and the artifacts that are employed for the propagation of information are ultimately what influence the effectiveness of handoffs. The system surrounding the task in turn structures the possibility to share and communicate information (Artman \& Garbins, 1998).

\section{Research Goals and Objectives}

Investigations into the environmental contexts of information flow in hospitals is especially important as there is a paucity of data published in the literature on handoff procedures and practices in the perioperative stages of care, such as patient stays in the recovery room (Kaafarani et al., 2009). The present research investigated current practices and procedures for patient care handoffs in the post-anesthetic care unit (PACU) of a large Ontario hospital. The research objectives were to understand and map the flow of information acquisition and dissemination along with the communication processes/dynamics between multidisciplinary clinical teams involved in patient care handoffs. Specifically, the research team was interested in mapping the distribution of 
communication among individuals in multidisciplinary teams and their tools/artifacts. The main focus for the future is to use the results to supplement previous findings and future research and develop procedures and/or suggestions that can mitigate potential communication breakdowns that lead to information loss. This goal will be accomplished by facilitating the conceptualization, design, and development of tools, devices, and technology for the support of handoff processes. With an enhanced understanding of how information propagates throughout various healthcare system environments, the chances of identifying design issues for future handoff support tools will be improved.

The researcher addressed the following questions:

- What is the structure of current handoff procedures?

- What topics of information are exchanged/conveyed during the transfer (medications, care plans, past medical history, etc.)?

- What medical personnel are involved in the handoff?

- What are the most common purposes for communication (e.g. to ask questions, confirm information, make announcements to the team, etc.)?

- What are the team interactions during handoffs (e.g. is the handoff a multiprofessional verbal interaction, does the team collaborate inter-professionally to detail the patient plan)?

The methodology employed followed a cognitive ethnography approach and in so doing, directed observations with the aforementioned research goals and specific data collection techniques (Patel et al., 2008) including interviews and field observations. The researcher gathered sufficient evidence to support preliminary suggestions for possible standardizations to content verbalized in handoffs. Through real-time observations and 
field notes the researcher obtained an in-depth understanding of the characteristics of handoffs; this knowledge contributed to the attainment of the study's objectives.

\section{Method}

The methodology employed in this study was primarily observational as the focus was placed on obtaining data on simultaneous interactions/communications and real-time processes in handoffs between multidisciplinary staff (anesthetists, nurses, surgery staff) from the OR to the PACU. A mixed-method approach was adapted in order to deepen the scope of understanding of the phenomena under study (Sandelowski, 2000). Interviews, ethnographic field notes from handoff observations, and complexity ratings from staff members were obtained and analyzed. The researcher was unable to control the observational environment and as a by-product of this research reality, patient cases were observed based on pre-determined criteria that included age $(18+)$ and the destination of the patient post-operatively. The primary participants were the medical and nursing personnel involved in the handoff but because of the ethnographic nature of the study, hospital patients were also within the observational environment.

\section{Study Sample}

Research into qualitative data collection itself revealed that large sample sizes are not necessarily required (Sandelowski, $1995 \& 2000$ ). The main importance in qualitative research is obtaining a sample size that can provide quality information for answering the research questions (Sandalowski, 1995). Prior to observations, a sample of 50 observations was deemed large enough to establish the types of information being conveyed during handoffs, along with the underlying structures (Foster-Hunt, 2009). The obtained sample size was motivated by the methodology employed and the data 
saturation required for the attainment of the goals of the study. At the point of 40 observations data saturation was reached as no new data (medical information categories) emerged. Past research has demonstrated that once new data does not appear and observations display recurring themes, the researchers can be fairly certain that data saturation has been achieved (Strauss \& Corbin, 1990).

\section{Patients}

In accordance with hospital regulations, patients were required to sign a consent form (see Appendix A) prior to observation. Patients were made aware that at any point in the research process they had the right to withdraw from the study with no penalty or risk of loss of quality and safety of care. Any patient at the hospital who was at least 18 years of age with the intended destination of the PACU post-operatively, and was able to sign a consent form or had a guardian who could sign a consent form on their behalf was eligible to be included in the study. Due to the aforementioned research criteria patients could not be randomly selected out of all those admitted on the day of scheduled observations.

\section{Healthcare workers}

Healthcare staff members who work in the PACU and the OR were passively consented for participation in this study. As such, all staff members who were involved with patient care handoffs and did not dissent to participate in the study were eligible to participate. The observed sample of healthcare workers included at least 1 anesthetist and 1 recovery room nurse per handoff but on average included 4.7 staff members.

Participation in this study was voluntary and participants received no benefits or rewards personally. 


\section{Data Collection Approach}

In this study, observations and data collection were guided by the research goals to understand current practices and procedures for patient care handoffs, to investigate the flow of information transfer and communication within a handoff and to potentially identify areas that might be susceptible to communication breakdowns. Investigating patient safety in handoffs through observations of communication patterns in multidisciplinary hospital settings allows for the visual observation of cognitively distributed tasks. When human behaviour or activity is observable between individuals and their tools/artifacts, ethnography is a useful tool for collecting qualitative data (Malhotra et al., 2007).

Data on the workflow of healthcare providers can be collected through cognitive ethnography, a combination of traditional ethnography and cognitive methodology (Hutchins, 2003). This approach to observing cognition within its social context was formulated on the bases of three principles drawn from ethnography: intensity, independence and personalization. Intensity is replaced by "specificity" of data collection, independence with "purposive" techniques (having specific research goals or theoretical underpinnings of the research) and personalization with "verifiability" (where the results must be validated across different settings) (Hutchins, 2003; Patel et al., 2008). Within the context of study, cognitive ethnography can be employed to understand "how system resources are configured and utilized to accomplish successful tasks and prevent adverse events" (Hazelhurst, 2000, p.539).

For the present study, cognitive ethnography was carried out through semi-structured interviews and staff shadowing during patient care handoffs. Through staff shadowing, 
the researcher collected data on the various features of teamwork in the PACU. These features might include verbal interactions, multi-disciplinary make-up of the care team and collaboration efforts. The researcher recorded the modes and methods of information exchange. Additionally, semi-structured interviews with three staff members from both anesthesiology and nursing were used to collect data concerning perceptions and expectations of handoff structure as well as the processes/procedures for handoffs. The data from the interviews validated the ethnographic observations and together, these data sources provided insight into the nature of handoff interactions in the PACU.

\section{Materials}

\section{Handoff observation form}

A paper-based tool developed by the researcher was used to capture data specific to the handoff. The development of this tool was guided by informal observations of handoffs in the recovery room (PACU). The researcher took notes on the type of patient/medical information that is conveyed during handoffs along with the typical length of handoffs, the personnel involved and the environmental context. The resulting tool cued the researcher to the specific information that was important to collect while acting as a checklist for noting several details such as: the dynamic of the handoff (i.e. multidisciplinary), the information conveyed (past medical history, drug information, patient orders etc.), the number of staff directly involved and the delivery method (verbal/nonverbal, asynchronous/synchronous) (see Appendix B). There was also a section for free-form handwritten notes where the researcher captured the communication exchanges throughout the course of the handoff (anything missed by the researcher was supplemented by audio recording). No personal health information was kept that could 
link the patient to the data and all data collected was stored in a locked cabinet in a locked office at Carleton University during the data analysis phase of the study and will be securely stored in the future as per the ethics approval requirements.

\section{Complexity ratings questionnaire}

A rating scale was used in order to rate the complexity of each patient case. The ratings were on a Likert scale from 1(not at all complex) to 5(highly complex) and were based on a subjective rating of the complexity of each patient's care post-operatively in the PACU. The name of each surgery was accompanied by any comorbidities (coexisting illnesses) that were presented in the verbal handoff report in order to assist the staff in completing reliable ratings and to ensure a high level of agreement (see Appendix C). Inter-rater reliability was calculated to ensure validity.

\section{Pre-observational interviews}

A semi-structured interview developed by the researcher was used for the interviews. This method was adapted in order to obtain as much detail as possible from the interviewees. The interview consisted of six open-ended questions concerning handoff details (e.g. "How do you perceive the safety of patient care at this hospital?" or " Describe the current procedure for a patient care handoff in this hospital"; see Appendix D)

\section{SBAR (Situation Background Assessment Recommendation) Protocol}

SBAR is a conceptual skeleton for checklists that was developed to improve communication among healthcare staff involved directly with patient care. The paper tool cues staff to the important pieces of information that should be shared amongst the team for various medical information categories. For example, for the 'situation' the care 
provider should identify themselves and their unit when calling about a patient, they should provide relevant 'background' information about the patient such as blood pressure, there should be an 'assessment' of what the problem is and finally, a 'recommendation' on what steps to take to resolve the problem. The SBAR "Background" checklist was used to assist in the coding process because it includes medical terminology and categorizations of medical information. Specifically, the Background section of the 'SBAR Report to Physician About a Critical Situation' form was used as a reference (see Appendix F).

\section{Data and Measures}

\section{Contextual factors}

This data included the documentation of verbal behaviours, staff involved, and technology used (e.g. computers, paper forms). The researcher was generally interested in the facets of communication between varying healthcare staff types involved in the handoff, e.g. is there a hierarchical working relationship present, is there verbal communication between professions, are these verbal communications clear and understood by the receiving party etc.

\section{Information flow/dissemination of information}

The researcher focused on the transfer of information during communication events in the handoff process. Specifically, the aim was to document the specific healthcare providers who transfer information. Information flow was evident through the representational states of information in a handoff. Data on information flow is presented in the analysis of speaker section in results. 


\section{Topics of information}

Information concerning medical history, current medications and dosages, treatments, and future care plans for medication and treatments were recorded. These specific pieces of information that are conveyed between healthcare workers are essential in outlining the structure of patient care handoffs. Furthermore, these details might give insight into how efficient and effective the handoffs are in terms of receiving and disseminating relevant patient information and ultimately how vulnerable they may be to communication breakdowns.

\section{Complexity of post-operative care}

The complexity ratings by staff members (detailed above) were collected with the intent of investigating whether post-operative care complexity would affect certain aspects of a handoff. Specifically, the researcher documented the relationship between post-operative care complexity and the verbal behaviours, types of medical information conveyed, duration, and the volume of communication observed.

\section{Handoff duration}

The length of each handoff was measured (in minutes) in order to investigate whether there was a relationship between duration and the other variables of interest such as complexity, medical topics discussed, and verbal behaviours.

\section{Procedure}

\section{Participant consent and recruitment}

Medical staff members were passively consented. Due to the nature of the present research, a large number of medical staff members were observed and therefore, all staff in the OR and the PACU were considered participants. Any staff member who had the 
potential to be involved in a patient handoff including all nurses in the PACU, anesthetists and assistants, physicians and porters were informed about the study prior to the commencement of observations. Multiple visits to the hospital units and to staff meetings on different days prior to observations served as a method for introducing the research study both informally and formally to the nursing staff. The researcher attended the anesthesiology morning rounds to introduce the study and address any questions or concerns by the medical staff. All staff members were informed that they were free, at any time during the study, to dissent from participation (there were no cases of staff members dissenting). This method of consent is approved by the research ethics board of the hospital in situations where there is no added risk to patients, families, or staff over the course of data collection. Participation in this research study was completely voluntary and as such participants were not rewarded for their involvement.

Due to the sensitive nature of the observational environment, the researcher was highly respectful and cautious during the recruitment and observation periods. The researcher was not directly involved with the patient. The only exception was for the purpose of consenting patients in the Pre-Anesthetic Unit (PAU) prior to their surgical procedures. In order to obtain consent prior to observations, the researcher arrived at the PAU the morning of the patient's scheduled procedure. The researcher was given permission by the manager of the PAU to view the surgical schedule on site on the day of observations. The researcher consented as many patients as possible for that particular day (as long as the patients met the research criteria) because observations were open to any surgical type. A PAU nurse brought an information card (which gave a brief summary of the research project) over to each patient who was to be involved in the study 
and asked if they were willing to be approached by the researcher. If the patient agreed, the researcher then approached the patient at their bedside and explained the study in greater detail. The researcher obtained written consent for those patients who agreed to have their handoffs observed (see Appendix A). If the patient was not able to provide consent a guardian signed the consent form on their behalf.

\section{Pre-observational interview}

A series of pre-observational interviews were completed with three members of each discipline involved in the transfer of patients (anesthetists and nurses). Healthcare staff (nurses and anesthetists) that participated in the interview were encouraged to provide as much information as possible in the allotted timeframe (maximum of 30 minutes). The researcher probed participants who did not provide detailed information. Audio recording was used to aid in the recording process, as there were no objections by any of the participants. The interviews lasted an average of 20 minutes.

\section{Observations of patient care handoffs}

One researcher from Carleton University recorded all of the handoff observations by hand onto the handoff tool (see Appendix B) while concurrently audio recording the handoffs. (It was announced to all staff members at the beginning of each handoff that the interaction was being recorded.) Any data that was missed in real-time by the researcher was supplemented by the audio recording. The researcher maintained close proximity to the interaction between healthcare providers during the handoff and removed herself from the patient's bedside once it was complete. During the course of observations the researcher did her best to avoid being overly intrusive in the observation of the patient's 
care and adjusted according to individual patient sensitivity to being observed in their vulnerable state.

\section{Administration of the complexity ratings questionnaire}

Once observations were complete the researchers contacted the nursing educator for the PACU in order to schedule a meeting with the nursing staff. At this meeting the researchers explained how to complete the complexity ratings and then asked six nurses to complete the ratings. At the same time, two experienced nurses (20+ years) were asked to verify the categorization of medical information that was gathered and organized during the analysis of the handoff observation data. To obtain ratings from the anesthesiology staff members, the staff anesthetist was contacted and given the questionnaire along with an explanation of how to fill it out. The staff anesthetist then collected three completed questionnaires from his staff members.

\section{Debriefing}

Participants could not be debriefed following a day of observations because several staff members were involved in multiple observations. Therefore, providing detailed information on the study might have biased participants or caused them to alter their behaviour. Upon completion of the study, the researcher scheduled in-hospital sessions to present the results to the healthcare professions involved in the study as well as to those with a vested interest in the findings (anesthetists, nurses, hospital administration). At this time the scope of the research was revealed and there was an opportunity for a discussion and/or questions from the staff. Contact information for individuals involved in the research was provided to all participants along with a written summary of the study. 
Results

The analyses presented here are descriptive and exploratory in nature due to the reality of qualitative data collection, especially within a hospital environment where random assignment and counter-balancing were not possible. As such, the majority of numerical data and relationships presented did not undergo statistical analysis (with the exception of a few non-parametric tests). General results of the characteristics of handoffs in the PACU including the description of the data coding approach along with the content and structure of handoffs according to observations and interviews will be presented first. Results detailing the characteristics of communication in observed handoffs relative to the variables of interest including case complexity, handoff duration, topics of information, and information flow. Finally, an analysis is presented regarding the use of resources in the PACU. Operational definitions applicable to each variable of interest will be presented in the relevant sections.

\section{Characteristics of Handoffs in the PACU}

Observed and recorded handoff data was transcribed verbatim (with identifiers removed to ensure anonymity) and then coded into content categories. The content categories were derived post-observation using background knowledge obtained from pre-observational interviews along with the SBAR protocol (see Appendix F) to guide the coding process.

\section{Information content categories}

The first step in the analysis was to identify information content categories that could later be used to identify the structure of handoffs and patterns in how information was shared (see Appendix E, Table 4, for a full list of content categories). Of the total 725 
observed discussions of medical topics, the majority of handoff information had to do with four general content categories: history, intra-operative events, patient status, and future care plan (see Table 1).

Table 1

Information Content Categories, Descriptions, and Examples

\begin{tabular}{|c|c|c|}
\hline Content & Description & Examples \\
\hline History & $\begin{array}{l}\text { Medical history, hemodynamic (blood } \\
\text { pressure, temperature etc), allergies, } \\
\text { comorbidities }\end{array}$ & $\begin{array}{l}\text { "Any allergies?" } \\
\text {-"Yes, she's allergic to } \\
\text { Codeine." }\end{array}$ \\
\hline $\begin{array}{l}\text { Intra- } \\
\text { operative } \\
\text { Events }\end{array}$ & $\begin{array}{l}\text { Any patient relevant event that occurred } \\
\text { in the OR, e.g., pain medications } \\
\text { administered, blood loss, treatments, } \\
\text { vitals, etc. }\end{array}$ & $\begin{array}{l}\text { "I gave } 100 \text { of Fentanyl in } \\
\text { the room." }\end{array}$ \\
\hline Patient Status & $\begin{array}{l}\text { Any patient relevant information (see } \\
\text { above for examples) at the time of the } \\
\text { handoff. }\end{array}$ & $\begin{array}{l}\text { "Can we give Tylenol for } \\
\text { the arm pain." } \\
\text {-"Yes, I would sooner do } \\
\text { that now than give } \\
\text { Fentanyl." }\end{array}$ \\
\hline $\begin{array}{l}\text { Future Care } \\
\text { Plan }\end{array}$ & $\begin{array}{l}\text { Any relevant information for the future } \\
\text { care of the patient. This can involve the } \\
\text { future care in the recovery room or the } \\
\text { surgical unit. }\end{array}$ & $\begin{array}{l}\text { "She's on Metoprolol and I } \\
\text { want her to get her next } \\
\text { regular dose." }\end{array}$ \\
\hline
\end{tabular}

The frequency of categories was calculated by counting each time that specific type of information (as described in Table 1) was discussed throughout the duration of the handoff. These numbers were then totalled across each of the 40 observations. The four categories combined accounted for $73 \%$ of the topics of information exchanges (see Figure 1), indicating that the majority of information sharing in PACU handoffs is concerned with these types of medical information. The most commonly discussed 
information category was history followed by intra-operative events, post-operative events and finally, future care plan. Overall, proportions of communications were fairly even among the four categories, although future care plan was discussed slightly less often. As there is no standard protocol that provides instructions on the specific information to convey, this finding demonstrates that healthcare staff involved in handoffs in the PACU recognize the importance of obtaining information in these specific medical categories. The next step in the analysis was to investigate handoff content to see if there were any commonalities in the structure of information within these four content categories.

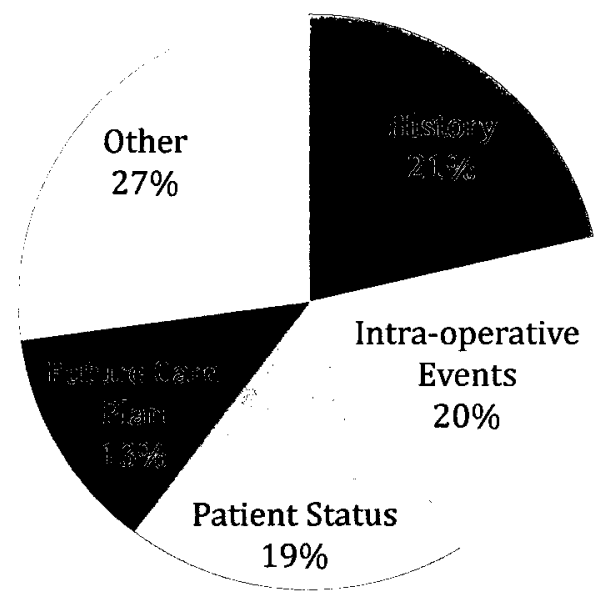

Figure 1. Frequencies of observed discussions involving specific content categories.

\section{Common handoff structures}

The following analysis was aimed at investigating the content of handoffs in terms of the chunking of information within content categories. Chunking is defined as the grouping of information that was verbally conveyed in the handoff. This included the sequence in which each content category was presented along with the grouping of 
related categories of information that were nested or embedded within (which will be referred to as sub-categories from this point forward). It is possible that the observed chunking may reflect the strategies healthcare staff employed for verbalizing relevant handoff information.

The structures were identified with the aid of preliminary coding (see previous section). A hierarchy emerged with respect to the manner in which patient-relevant details were verbally presented. The four general categories (see Table 1) were often accompanied by recurring sub-categories of information. For example, 'intra-operative events' was one of the four categories and one of the embedded sub-categories was 'pain management' which could then be followed by 'drug dose' and/or 'drug name' (see Table 2). Similarly, 'future care plan' as a category could also have these exact subcategories embedded within.

Table 2

Examples of the Structure of Content Categories

$\begin{array}{lll}\text { Information Provided General Content Sub-Category } & \text { Sub-sub Category }\end{array}$ (Nurse or Anesthetist) "100 of Fentanyl Intra-operative Pain Management Drug Dose and given in the room." Events Drug Name

The analysis identified a common structure for grouping medical information. Figure 2 details the common structures that consistently emerged in the observed handoffs. The figure contains acronyms used in the coding process including Cardio-Vascular System (CV), Genitourinary System (GU), blood oxygen saturation (SATS), and arterial line (ART). 
The analysis implied that anesthetists use common chunking strategies for grouping medical information. Although the verbal order of presentation of the main categories themselves over all the observed handoffs did not emerge as a common structure, the embedding of sub-categories within the general categories implies a common inner content structure for reporting information in handoffs in the PACU. General and subcategories were the focus of the analysis to follow.

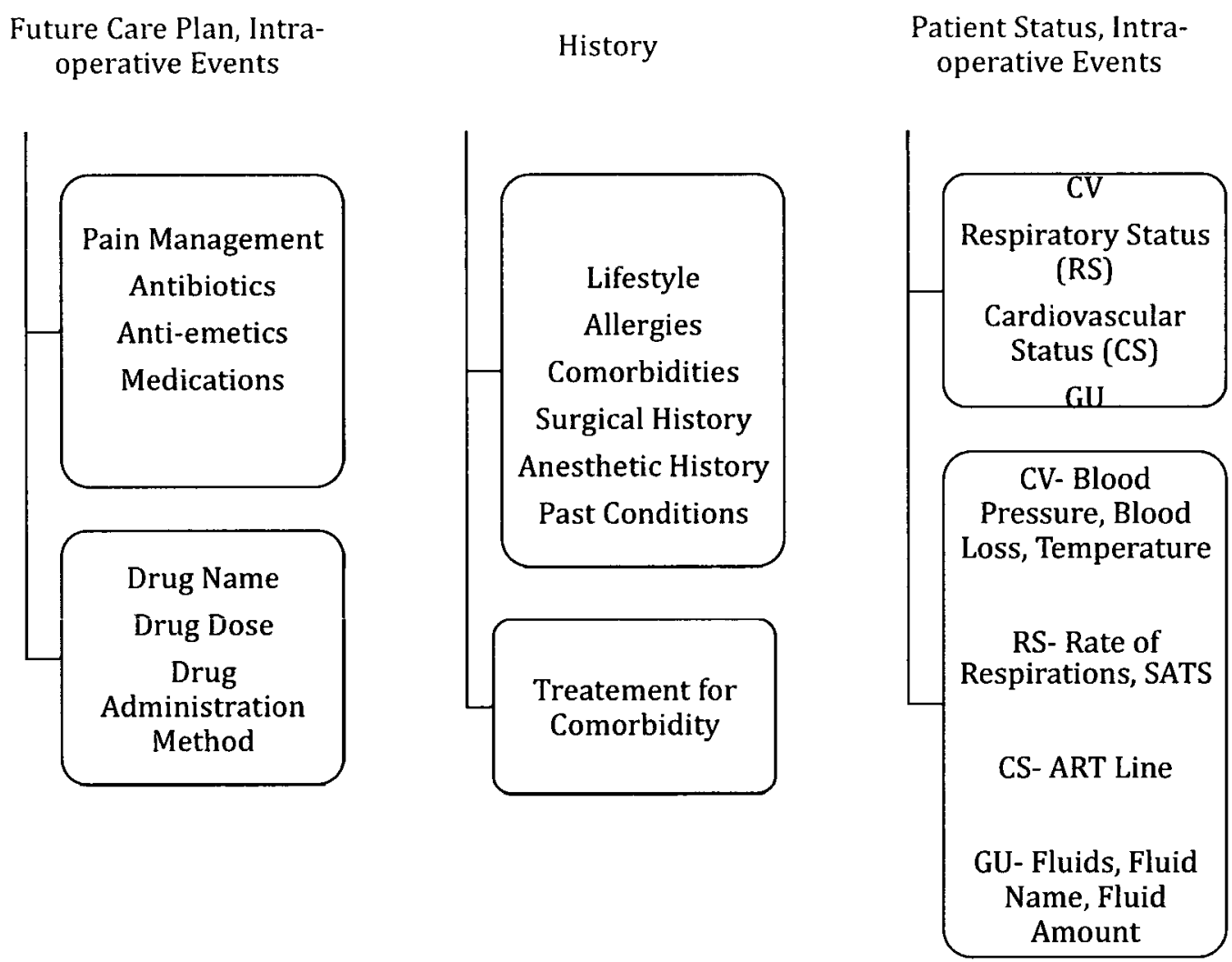

Figure 2. Flow chart detailing the common informational structure that emerged from handoff observations. The figure also displays content category names (general category, and sub-categories) and the breakdown of information content reported by anesthetists. The three content levels in the flow chart details the hierarchy of information. 


\section{Suggested handoff structures}

In order to further investigate the information structure that emerged from the observational data (see Figure 2), the pre-observational interviews were analyzed. Healthcare staff members who are directly involved in handoffs in the PACU were asked to identify the information content and order of presentation that should be conveyed in a handoff. The interview data underwent a similar analysis to that of observational data coding where the researcher used the same content categories to code the information and then looked for any implied structures between the categories that were identified. The structures that emerged from the interviews were then used as a benchmark for comparison with the observational data. The intent was to uncover whether the suggested structure matched the observed structure.

According to anesthetists and nursing staff, there are four major medical information categories that should be presented verbally in a handoff. Those four categories are: 1) history, 2) intra-operative events, 3) patient status, and 4) future care plan. (Demographic information identified by staff was grouped into the category of "demographics" for the purposes of the comparative analysis to follow.) Each of the four categories had accompanying embedded medical information that was deemed as important to present according to the healthcare staff members who were interviewed (see Table 3).

Table 3

Suggested Content Structures According to Pre-observational Interviews

Main Category

Demographics
Mid-Level Category

Patient Name

Patient Age

Procedure

Type of Anesthesia 


\begin{tabular}{ll} 
& Comorbidities \\
\hline Intra-operative Events & Antibiotics \\
& Pain Management \\
& Blood Loss \\
& Anti-emetics \\
\hline Patient Status & Vitals \\
\hline Future Care Plan & Pain Management \\
& Anti-emetics \\
& Antibiotics \\
CV (cardiovascular system) \\
Patient Discharge Information \\
Confirm nurse does not need anything \\
else
\end{tabular}

\section{Gaps between suggested and actual information structures}

In order to look for possible gaps between what should be conveyed in a handoff and what was actually conveyed, the researcher looked for a match between the content structure suggested by interviewees (see Table 3 ) and what was verbally presented in the observed handoffs. A value of 1 was assigned to content from the observed handoff that matched suggested content, whereas a value of 0 was assigned when there was no match between observed and suggested content; this was completed for each of the 40 handoffs. A "match score" was computed by adding the frequency of 1 s for the suggested categories overall, as well as for each individual category (demographics, history, intraoperative events, patient status, and future care plan). Descriptive statistics were then calculated.

The highest possible match score overall was 20 , which would result if all of the suggested information topics within each of the main categories (total of five) were presented in the handoff. According to the analysis, the mean match score was $8.5, S D=$ 2.9 with a mode of 8 , suggesting that more than half of the suggested information was not 
actually verbally presented during handoffs in the PACU. For the individual categories, the results were as follows: demographics (out of a possible high score of 4 ) $M=2.6$, history (out of a possible high score of 2) $M=1.6$, intra-operative events (out of a possible high score of 4) $M=1.4$, patient status (out of a possible high score of 3 ) $M=1.0$, and future care plan (out of a possible high score of 6) $M=1.9$. These results indicate that there is a gap between staff perceptions of what should be done and what is actually done in the handoff.

\section{Case complexity}

The post-operative care complexity ratings by nurses and anesthetists were analyzed separately for inter-rater reliability. Both separate ratings were reliable according to the intra-class correlation statistic, nurses $=.93, p<.01$ and anesthetists $=.80, p<.01$, respectively, as well as when a mean rating was calculated from the combination of both staff type's ratings, all $=.94, p<.01$. The remainder of the analyses to follow used the complexity ratings produced by anesthetists because they were the healthcare staff members who guided the structure of the handoff. The results did not allow for a grouping of high and low complexity handoffs because there was too much observed variation in the scores $(M=2.9, S D=.97, \operatorname{Mode}=3, \operatorname{Min}=1, \operatorname{Max}=5)$. Therefore, the relationships between complexity and the variables of interest (e.g. verbal behaviours, duration, information categories, etc.) were investigated as a function of each of the five levels of the scale.

\section{Handoff duration}

Handoff duration was measured using the audio-recorder that was started as soon as the patient was wheeled into the bed bay and stopped when the anesthetist giving the 
report left the patient's bedside. The researcher observed a total of 215 minutes of handoffs. Handoff durations ranged from 1.5 minutes to 16.1 minutes $(M=5.5$, Mode $=$ 3.1). There was no significant relationship between handoff duration and complexity, Pearson's $r=.25, p>.05$, implying that more complex cases did not necessarily entail longer handoff durations. (One outlier did not involve any verbal communication.) Characteristics of Communication in Handoffs

Conversation analysis adapted from Parush et al. (2009) and Pomerantz \& Fehr (1997), guided the mapping and description of communication patterns. Verbal interactions from observed handoffs were segmented into utterances and the resulting unit of analysis was each utterance produced by a healthcare staff member in the PACU. Utterances were not always between multiple staff members, e.g. an announcement to the team, "Fentanyl is in". The example below shows a sequence of utterances between a nurse and an anesthetist (belonging to the content category of future care plan).

Nurse to A: What about giving her a Tylenol suppository?

A to Nurse: I would sooner do that than give Fentanyl.

Analysis of the number of utterances best described the volume of communication observed. There were a total of 942 utterances (excluding the outlier handoff) observed over 40 handoff observations $(M=24.54$ per handoff, $\operatorname{Mode}=23$, Min $=11, \operatorname{Max}=50)$. Data was analyzed relative to handoff duration and complexity, information content categories, verbal behaviours, speaker, and resource consulted. 


\section{Communication and handoff duration}

There was a positive correlation between the number of utterances and handoff duration, Pearson's $r=.62, p<.01$. Therefore, as the number of utterances in a handoff increased so too did the duration of the handoff (see Figure 3).

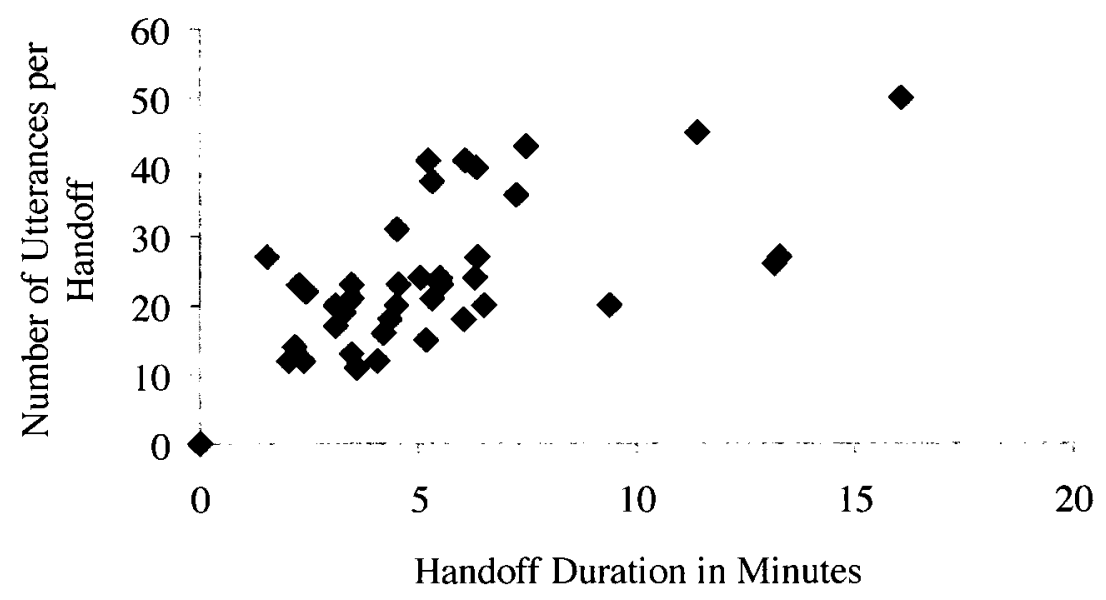

Figure 3. Relationship between the number of utterances per handoff and the handoff duration.

\section{Communication and handoff complexity}

The complexity of a handoff was positively correlated with the number of utterances observed, Pearson's $r=.41, p<.01$. Therefore, as the complexity of the handoff increased 
the total number of utterances in the handoff also increased (see Figure 4).

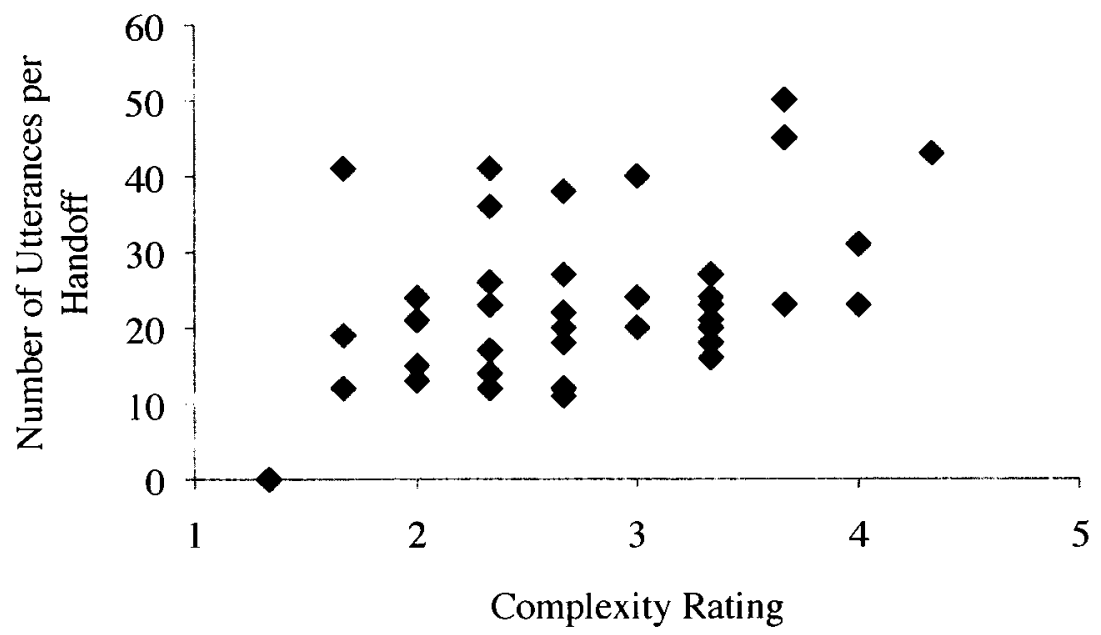

Figure 4. Relationship between the number of utterances per handoff and the complexity of each handoff.

\section{Verbal behaviour types}

Categories describing the type (e.g., purpose) of communication were based on conversation analysis adapted from Pomerantz \& Fehr (1997), Parush et al. (2009), and Kramer (2009), combined with categories that emerged through data coding. This method produced eight high-level categories: seeking information, responsive information, offering information, proactive information/report, requesting information, educating, comments, and irrelevant information. Each high-level category contained several subcategories (17 sub-categories in total). Table 4 summarizes all of the high level and respective sub-categories of communication types, each illustrated by an example (adapted from Kramer, 2009). 
Table 4

Verbal Behaviour Categories, Descriptions, and Examples

High Level Category Sub-Category $\quad$ Example

Seeking Information

One person requires information

from another.

\section{Question:}

Requesting information known (or assumed to be known) by another person, such as a numerical value or current state of affairs

Clarify:

Asking for further information to elucidate a previously

provided response

Anticipate:

Asking a question in which the answer is already expected, desired, or implied
"What did the patient get

for nausea and

vomiting?"

"What did you say to give him now for pain?

Fentanyl or

Hydromorph?"

"Ready to turn?"

\section{Proactive Information/ Report}

Providing information without being prompted. Solely an anesthetist verbal behaviour.

\section{Responsive Information}

Providing information in response to another type of communication

\section{Reply:}

Providing previously unknown information needed by the requester

\section{Confirm:}

Acknowledging that a request was made and indicating the manner that it will be acted upon

\section{Read-back:}

Repeating verbatim the words of the requester

\section{Offering Information}

Information offered without having been requested or sought by another
Announce:

Offering information to other members about the current state of affairs without being asked
"This is patient $\mathrm{X}, 68$ years of age, no past history to report."

"What time did he get his first dose of antibiotics?" -“10. Sorry, 9:30."

"Let's put the patient on their side."

-"Okay."
"You got the temp I gave you? 36.3." -“36.3."

"There's no need for antibiotics." 
Plan:

Similar to announcements, but indicative of future events

Concern-Raising:

Alerting the team to current or possible problems

Brief:

Bringing someone "up to speed"
"She is on Metoprolol so we'll need to give her next dose in 2 hours."

"I think there is something wrong with the stent."

"This patient has a history of airway issues, has a latex allergy and was consented for epidural."

"More saline please."

For objects:

Asking for a tool or a piece of equipment

For actions:

Directing another to do something

\section{Educating}

Instructing, guiding, or quizzing.

\section{Coach/Teach:}

Assisting during a situation where that person was not yet proficient
"Do you want to give him some IV Toretal or do you want to wait until he's awake and give Celebrex?"

\section{Commenting}

Extending a previous

communication without providing new information to the situation

\section{Discuss/Explain:}

Case relevant communication used to elaborate on a previously mentioned topic

Other:

Case relevant information, but not new or needed

\section{Irrelevant}

Content and intent had nothing to do with the current case or other patients
Joke

Non-serious, or sarcastic nature, light-hearted, laughable conversation

\section{Socialize}

Case-irrelevant, personoriented conversation
"Also since this one doesn't work with our unit, we can't use it..."

"I should be wearing gloves."
"How come you get so much vacation time doc? I never leave this place!" -"haha."

"Did you have a good weekend?" 
The distribution of verbal behaviour types in Figure 5 demonstrates the most frequently occurring high-level categories of verbal behaviours (see Table 4 for list and descriptions). Reporting information was the most frequent verbal behaviour type (44.4\%) followed by responsive information (20.1\%), offering information (17.1\%), and seeking information (14.7\%); these behaviours combined accounted for approximately $94 \%$ of communications. These results indicate that the largest proportion of communication is proactively offered by anesthetists (as only their utterances were coded as 'report') and the remainder of frequent verbal behaviours involved information sharing, receiving, and requests. (See Appendix E, Table 1, for the complete data set.)

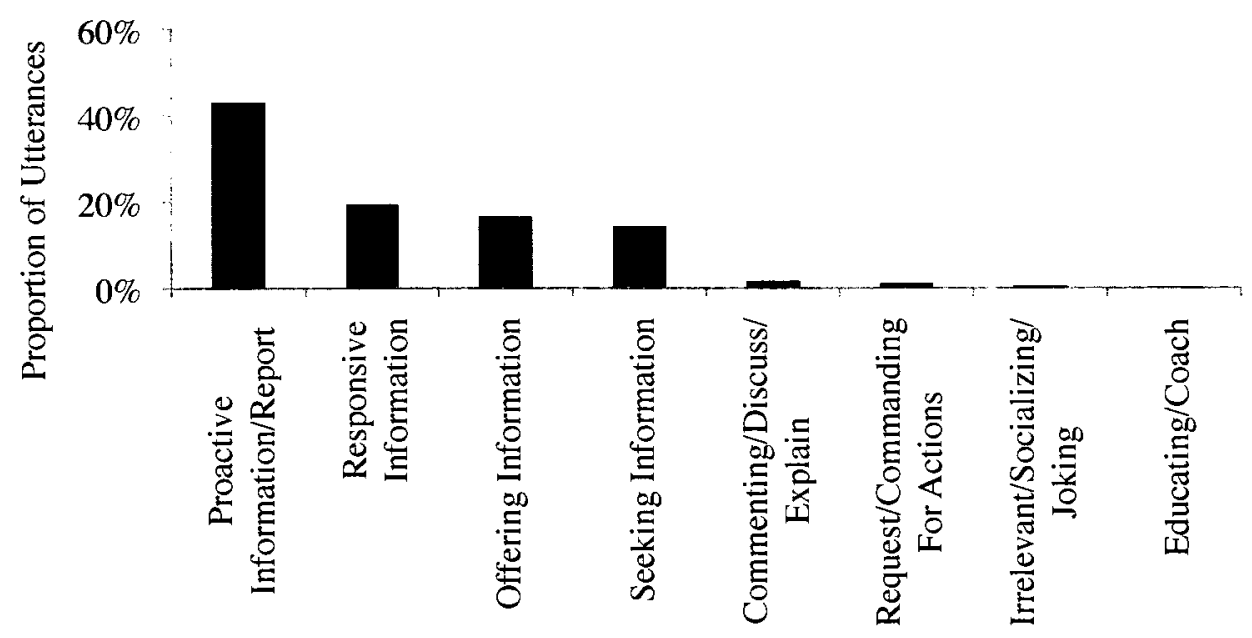

High-Level Verbal Behaviour Category

Figure 5. Frequencies of high-level verbal behaviour categories relative to the total number of utterances.

Analysis of the sub-categories of verbal behaviour types (see Figure 6) indicated that questions ( $12.3 \%$ of utterances) and replies ( $15.3 \%$ of utterances) were the most frequent communication types for healthcare staff to engage in. This result implies a reasonable proportion of closed-loop conversations when it comes to seeking information because of 
the similar proportion of questions and replies. The next most common reasons for communicating were to brief, announce, confirm, and to plan (see Appendix E, Table 1, for percentages). Communication coded by type was further analyzed as a function of information structure and content categories, speaker, resource consulted, complexity, and duration.

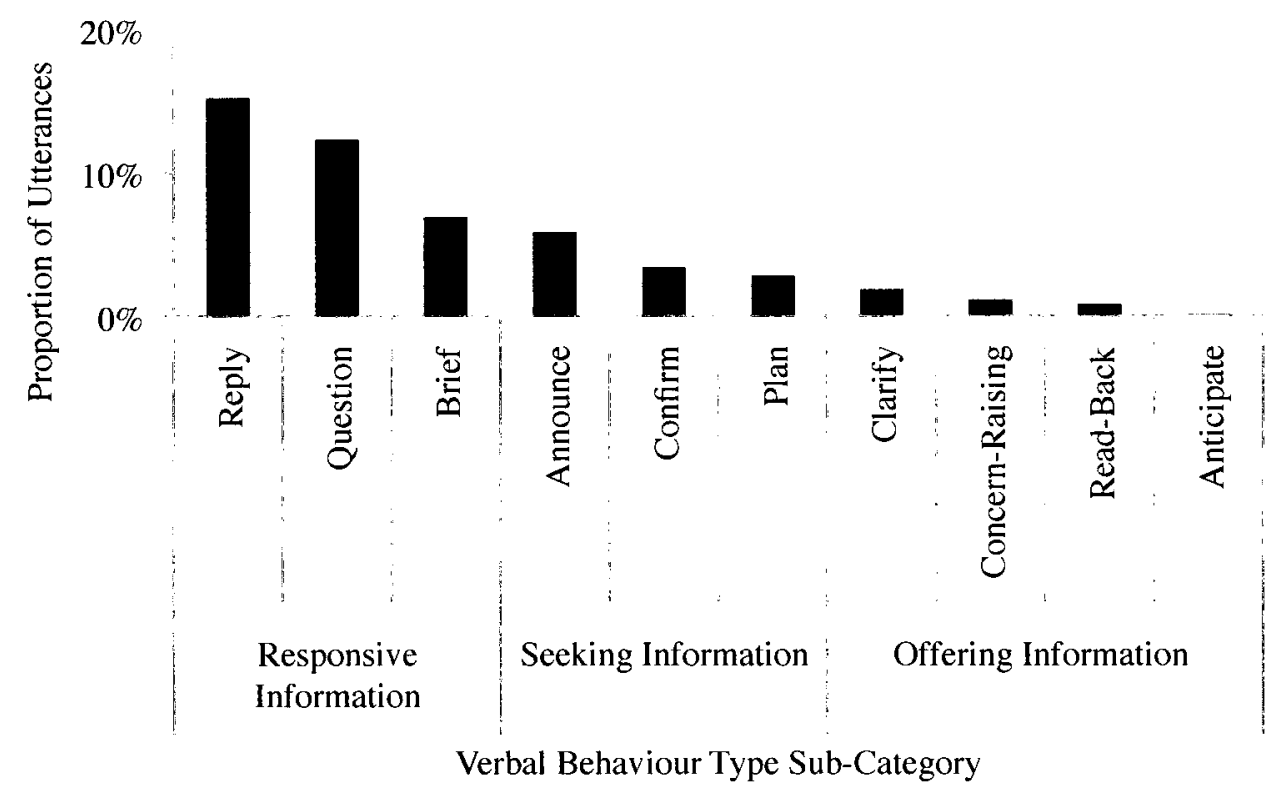

Figure 6. Frequencies of verbal behaviour type sub-categories relative to the total number of utterances.

\section{Relationships Between Suggested Structure and Variables of Interest}

Correlations were computed in order to investigate the relationships between each handoff's overall match score (see 'suggested handoff structure') and verbal behaviours, complexity, and duration. The aim was to uncover whether increased complexity, duration, and instances of verbal behaviour types were related to a match with the suggested topics. There was a weak positive relationship between the match scores and responsive information, $r=.33, p<.05$, a moderate positive relationship with offering 
information, $r=.45, p<.05$, and no significant relationship with seeking information, $r=$ $.30, p>.05$. These results indicate that the frequency of information exchanges increased as the match score increased however, not when it came to seeking information (questions for the most part, $r=.29, p>.05$ ). A strong positive correlation with report giving, $r=.79, p<.01$ serves to confirm the validity of the analysis, as it is apparent that an increase in reporting by anesthetists was associated with a higher match score for each handoff. Interestingly, an increase in case complexity was not related to a higher match score, $r=.20, p>.05$. This result may imply that the complexity of a patient's postoperative care does not necessarily cue healthcare staff to cover as many categories of information as possible. There was also no significant relationship between match scores and the duration of the handoff, $r=.25, p>.05$, indicating that more time available for interaction did not necessarily result in the handoff team discussing a wider variety of patient related details.

Verbal Behaviour Types and Information Categories

In addition to uncovering the most frequent reasons for communication and types of information exchanged, the research intended to investigate which high-level verbal behaviour types (see Table 4) were most frequently associated with specific information categories in handoffs. An analysis was performed on both variables relative to the total number of utterances for each verbal behaviour type. The following analysis (see Figure 7) focused on the four most frequently discussed information categories, which included: history, intra-operative events, patient status, and future care plan (aforementioned categories were also identified in the preliminary interviews). 
Report giving was a more frequent verbal behaviour for the information categories of patient history ( $42.6 \%$ of utterances) and intra-operative events (39.7\% of utterances). Comparably, when patient status ( $8.5 \%$ of utterances) and future care plan $(9.2 \%$ of utterances) were the focus of discussion, it appears that the frequency of report giving dropped proportionately. Seeking information was a much more frequent verbal behaviour when discussing patient status ( $46.6 \%$ of utterances) and future care plan ( $29.5 \%$ of utterances), whereas the frequency of these verbal types was much lower for intra-operative events (18.9\% of utterances) and history (5.3\% of utterances). It appears that report giving decreases as the instance of seeking information increases, which is visible for the patient status and future care plan information categories (see Figure 7). Responsive information was most frequent when communication involved the information categories of patient status ( $50 \%$ of utterances) and future care plan ( $28.5 \%$ of utterances) and corresponds with seeking information being the most frequent verbal behaviour for these two categories, and demonstrates a positive pattern of communication. Offering information was also most frequent for patient status $(30.2 \%$ of utterances) and future care plan (31.9\% of utterances) information exchanges. The aforementioned verbal behaviour types (seeking information, responsive information, and offering information) were less frequent for the information categories of history and intra-operative events because report giving composed $79 \%$ and $62 \%$ of utterances respectively. This result might suggest that the latter two information categories (patient status and future care plan) have more interactive points of information transfer as they are composed of a variety of interactive verbal behaviour types. 


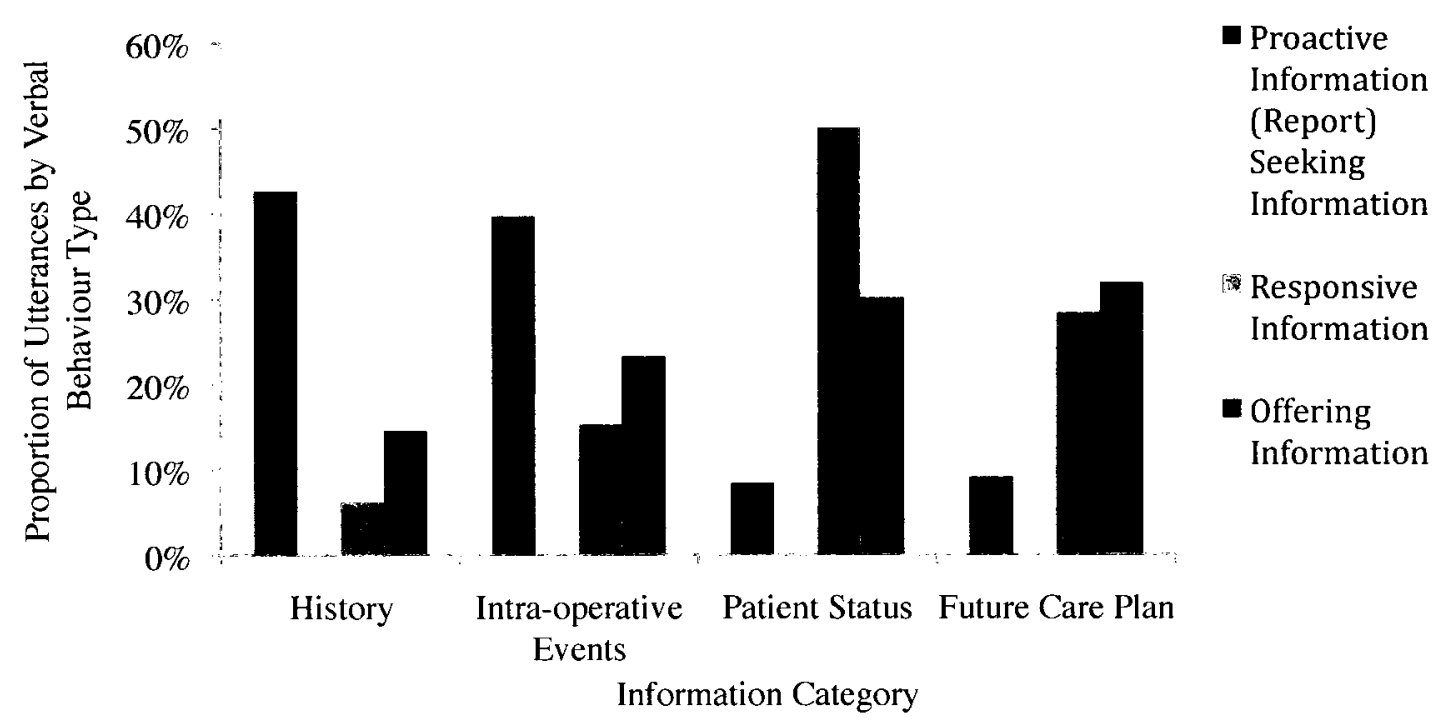

Figure 7. Frequencies of verbal behaviour types as a function of the information category and relative to the total utterances for each verbal behaviour type.

\section{Question, reply, and report as a function of information categories}

Due to the fact that questions and replies were the most frequently occurring subcategories of verbal behaviours (see Figure 6), an analysis was performed to investigate which information categories these behaviours were more frequently associated with (see Figure 8). As mentioned previously, seeking information instances increased when discussing the patient status and future care plan information categories compared to the other two categories (history and intra-operative events). The present analysis demonstrated that this occurred because the number of questions (measured across all four information categories) was proportionately larger in the patient status ( $46.4 \%$ of all questions) and the future care plan (32.1\% of all questions) information categories than in the history (3.6\% of all questions) and intra-operative events (17.9\% of all questions) categories (see Figure 8). 


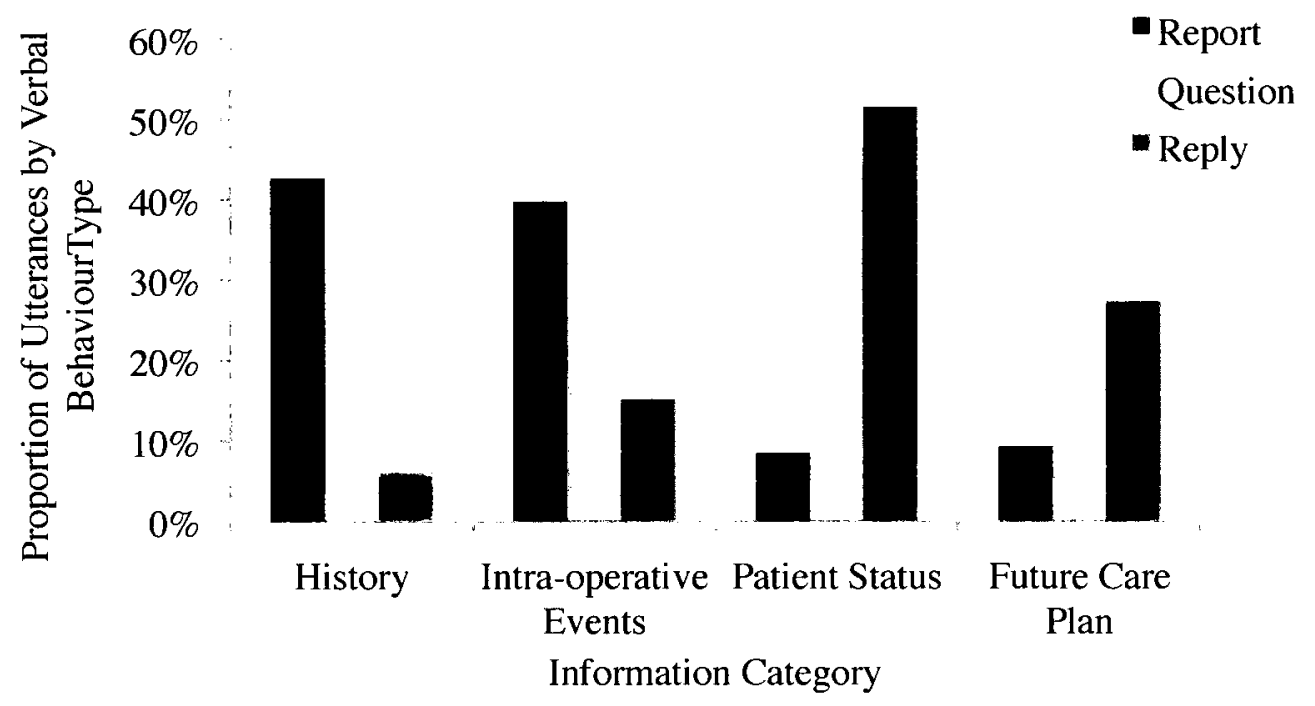

Figure 8. Frequencies of questions, replies, and reports as a function of the information category and relative to the total number of utterances for each verbal behaviour type over the four information categories.

There appeared to be a relationship between a decrease in report giving and an increase in the number of questions asked (see Figure 8). However, the relationship between the two variables was non-significant, $\chi^{2}(3, N=356)=2.13, p>.05$. Analysis of speakers

Over the course of observations the researcher documented various healthcare workers from different disciplines who were involved in handoffs including nurses, anesthetists, personal care assistants, respiratory therapists, and surgery staff. For coding purposes, there were three categories of speakers: nurses (RN), anesthetists, and 'other' (surgery, personal care assistants, respiratory). In order to assess the dynamics of communication between the handoff team members, the proportion of speaker-specific communications relative to the total utterances over the course of observations was 
analyzed (see Figure 7). Utterances by anesthetists (72.0\%) and nurses (26.1\%) comprised the majority of communications (approximately $98 \%$ of utterances) whereas 'other' types of staff accounted for a very small proportion of utterances (1.9\%).

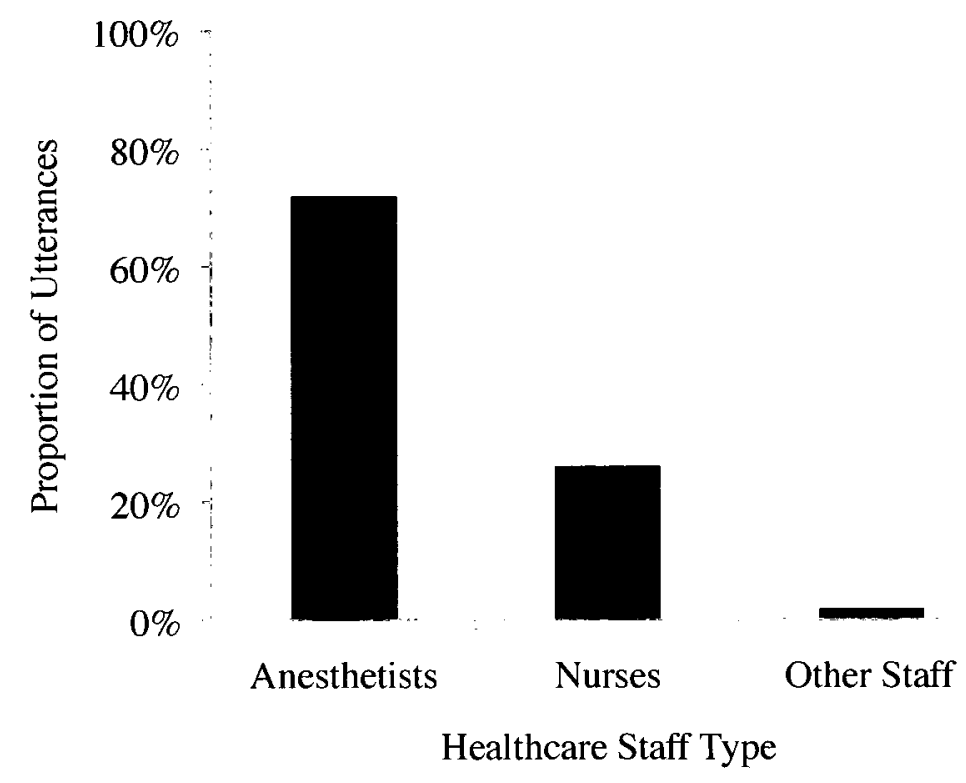

Figure 9. The proportion of total utterances relative to healthcare profession.

Speakers and verbal behaviour types

The analysis to follow is supplementary to previous results (see Figure 5) that reported on the frequency of verbal behavior types relative to the total observed utterances (942). In addition to those results, the present analysis explored which speaker type was most frequently associated with the specific verbal behavior type. Report giving by anesthetists was the most frequent form of communication in handoffs $(42.7 \%$ of utterances) whereas nurses were associated with a very minimal proportion of utterances of this nature ( $0.3 \%$ of utterances). Responding to requests for information was the next most frequent verbal behaviour for both anesthetists and nurses. When it came to seeking 
information, nurses engaged in this verbal behaviour more frequently than anesthetists ( $10.8 \%$ of utterances to $3.7 \%$ respectively). The similar rate of nurses' seeking information utterances compared to anesthetist response utterances ( $10.8 \%$ for both) demonstrates a positive pattern of communication where closed-loop conversations are more frequent than open-looped (see Figure 10; see Appendix E, Table 2, for percentages).

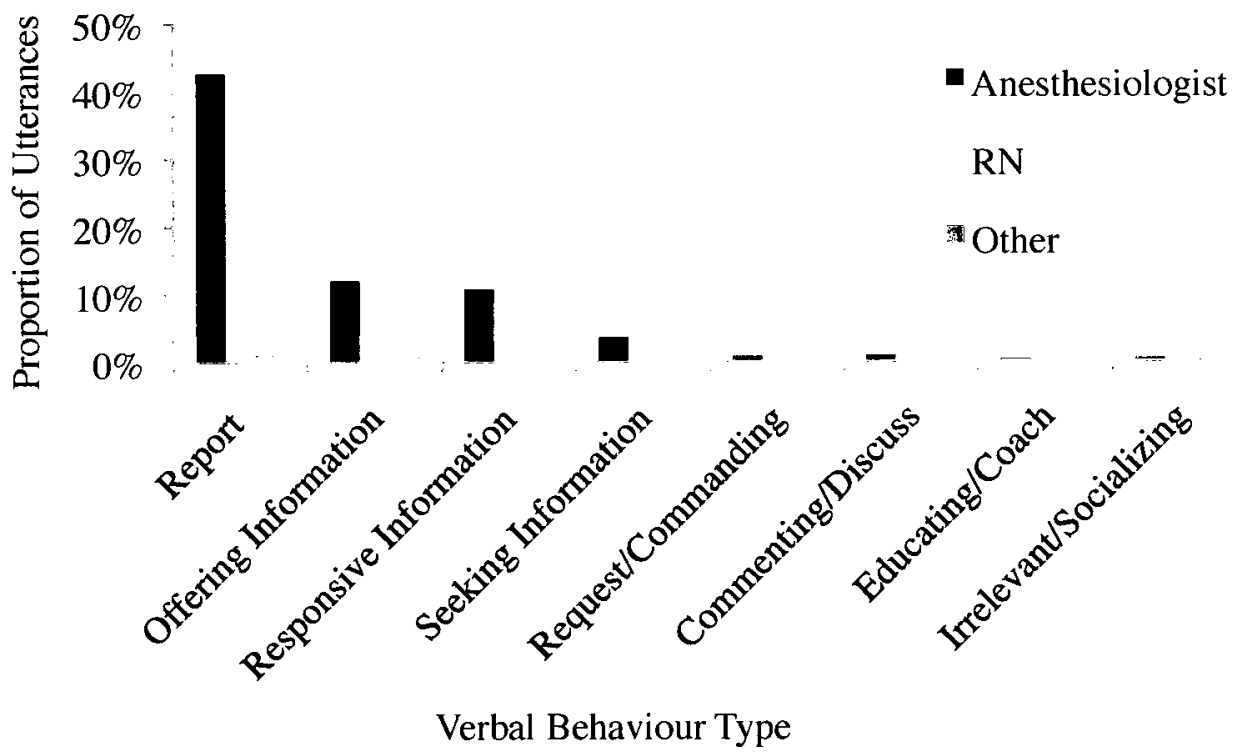

Figure 10. Speaker-specific verbal behaviours relative to the total number of utterances.

\section{Seeking information and speaker}

The next step was to identify which healthcare staff members were associated with the majority of questions, as this was the most common sub-category (see Figure 6) of verbal behavior types. The analysis was focused on the patient status and future care plan categories because in comparison to the other two categories (history and intra-operative events) report giving dropped proportionately whereas the frequency of questions increased. The intention was to investigate the relationship between question and replies 
relative to the speaker for these two information categories. The results of the analysis indicate that nurses are asking the majority of questions in both information categories ( $74.4 \%$ of questions concerning patient status and $78.6 \%$ of questions concerning future care plan; see Figures 11 and 12). Along with the fact that report giving is quite low for these two information categories, this is an interesting result because in interviews nurses explained that they know ahead of time what information they need to get from anesthetists during the handoff. A direct quote taken from an interview with a PACU nurse exemplifies this well; "Umm I think handoffs are safe in that the nurses are experienced enough to ask questions if they're not happy with the lack of information or lack of report. They know what questions to ask". The relationship between questions and replies related to the patient status category and as a function of the speaker was significant, $\chi^{2}(1, N=89)=8.73, p<.05$. For the future care plan category, the relationship was also significant, $\chi^{2}(1, N=54)=16.76, p<.01$. For both information categories, the nurses asked significantly more questions and the anesthetists had significantly more replies. 


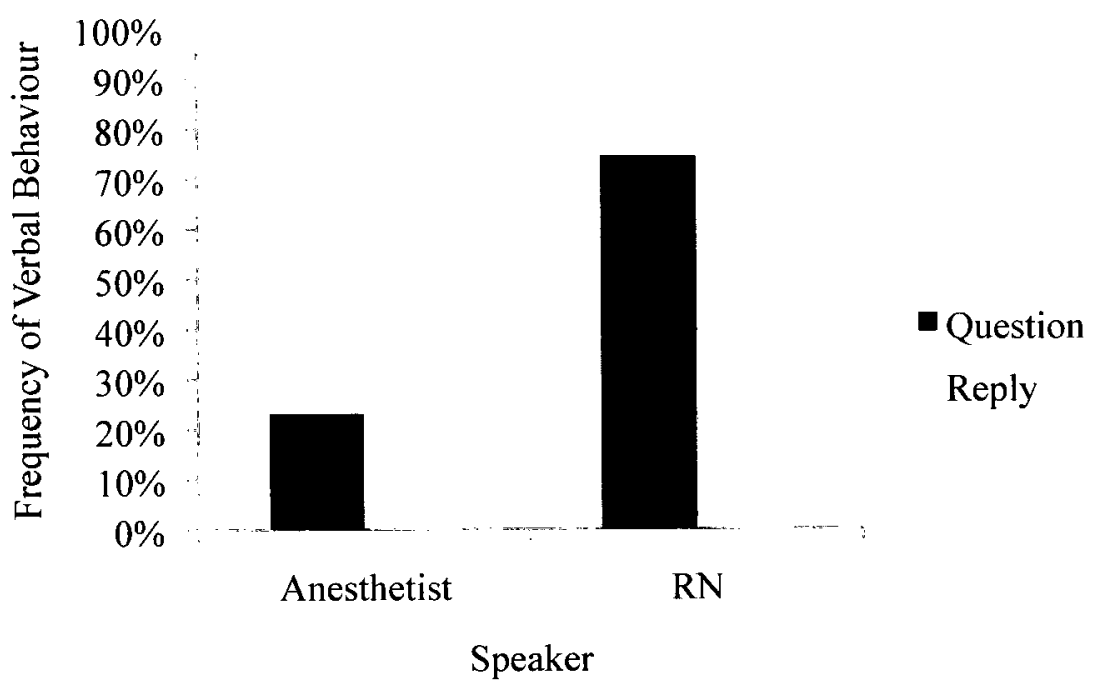

Figure 11. The frequency of questions and replies by speaker for the information category of patient status relative to the total number of utterances for that verbal behaviour type.

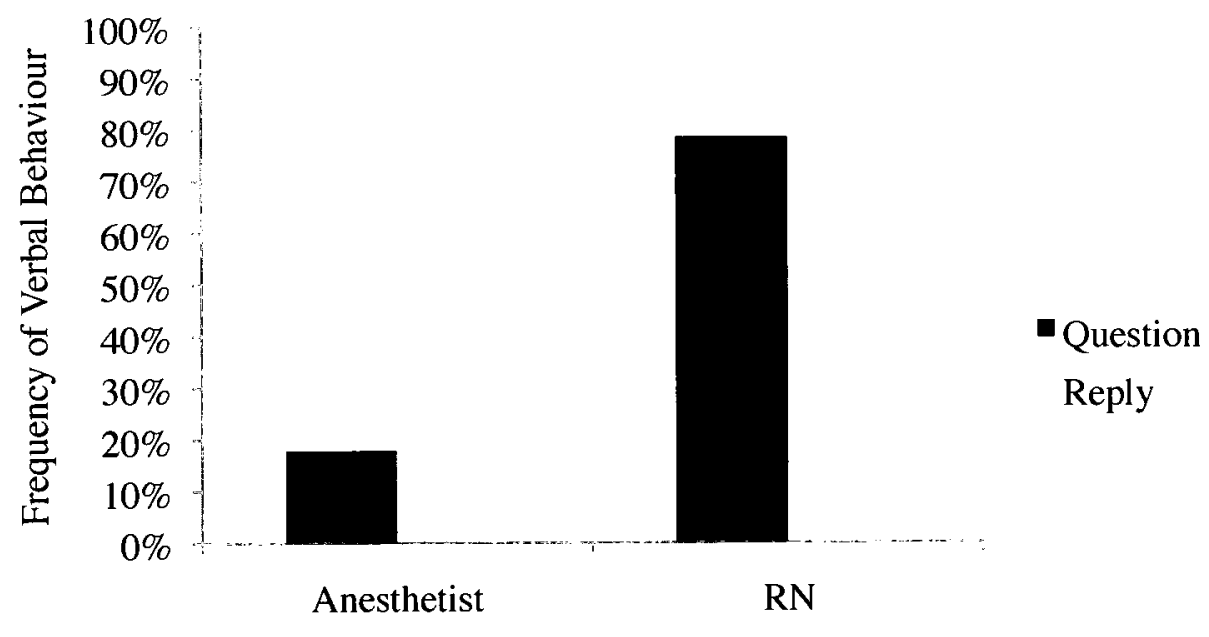

Speaker

Figure 12. The frequency of questions and replies by speaker for the information category of future care plan relative to the total number of utterances for that verbal behaviour type. 


\section{Use of Resources}

A resource in the PACU is any tool that can be used to access or record medical, patient relevant information (hand filled forms, computers, etc.). During the course of observations, the researcher made note of the various resources that were consulted during handoffs. The use of a computer program to document handoff information became a variable of interest as this tool was introduced into the PACU just past the midway point of observations. In observations that did not involve a computer, nurses always recorded handoff information on a PACU flow sheet. This sheet was only used by nurses in the Post-Anesthetic Care Unit for taking notes on the patient and their vitals; the front-side has spaces to record medications and dosages and the back-side covers a headto-toe assessment of the patient. This is not a formal document and thus, it is not required to be filled out and is not a part of the patient chart.

\section{Use of computer program during handoffs}

The introduction of a handoff computer aid called 'Periop Manager' into the PACU during observations allowed the researcher to analyze data relative to the use of this resource. Periop Manager is a documentation program intended for use throughout the operative process of a patient, all the way from the pre-operative visit to the postoperative care. In the PACU only the nurses use the computers and although there are expected fields of completion such as: name of person giving the report, the surgical procedure, patient assessment, neurological assessment, vascular assessment, invasive lines, vital signs, dressings, medications, fluids, regional/spinal anesthesia, ventilator settings and trends, there are no mandatory completion fields. The program inserts markers for the time of admission to the PACU, the time ready for discharge, and the 
actual discharge time. There are spaces to document other issues such as sleep apnea, IV pain control, discharge details, and personal notes.

Post-observation, handoff data was separated into two groups: one group that did not involve the use of a computer for 24 handoff observations (called "No Computer") and another that involved the use of a computer for 15 handoff observations (called "Computer"). Handoffs observed without the use of a computer and with the use of a computer occurred in chronological order, meaning that the Periop Manager program was introduced on the 25 th observation. The outlier handoff was removed from the following analysis.

The average duration of observed handoffs did not differ greatly with the introduction of the computer program $(M=5.4$ minutes without computer; $M=5.6$ minutes with computer). Likewise, the average number of different high-level categories (out of a possible 13$)$ discussed did not vary at all $(M=7.9$ topics without or with a computer $)$. The mean number of utterances per handoff increased by three once the computer program was introduced ( $M=23$ without computer; $M=27$ with computer) and the total number of verbal behaviours ( $M=18$ without computer; $M=20$ with computer) increased on average by two per handoff. On average, healthcare staff covered one more suggested information topic (an increase in the match score) per handoff than was covered without use of the program ( $M=8$ without computer; $M=9$ with computer). These results might indicate a level of consistency across handoffs despite the introduction of the computer aid. 
Verbal behaviour types with and without use of the computer program

An analysis was performed in order to compare the frequency of verbal behaviour types with or without use of the computer program relative to the number of utterances observed for each type of behaviour (see Figure 13). Reporting, questions, replies, concern-raising, and briefing were more frequent without use of the computer program. Clarifying, confirming, and planning were more frequent with use of the computer program (see Appendix E, Table 3 for percentages). It appears that computer use might have encouraged several verbal behaviours that look for further elaboration. (There was only one utterance overall to anticipate information and a very low frequency of educating, commenting/discussing, and irrelevant information, so those verbal behaviour types were omitted from the present analysis.)

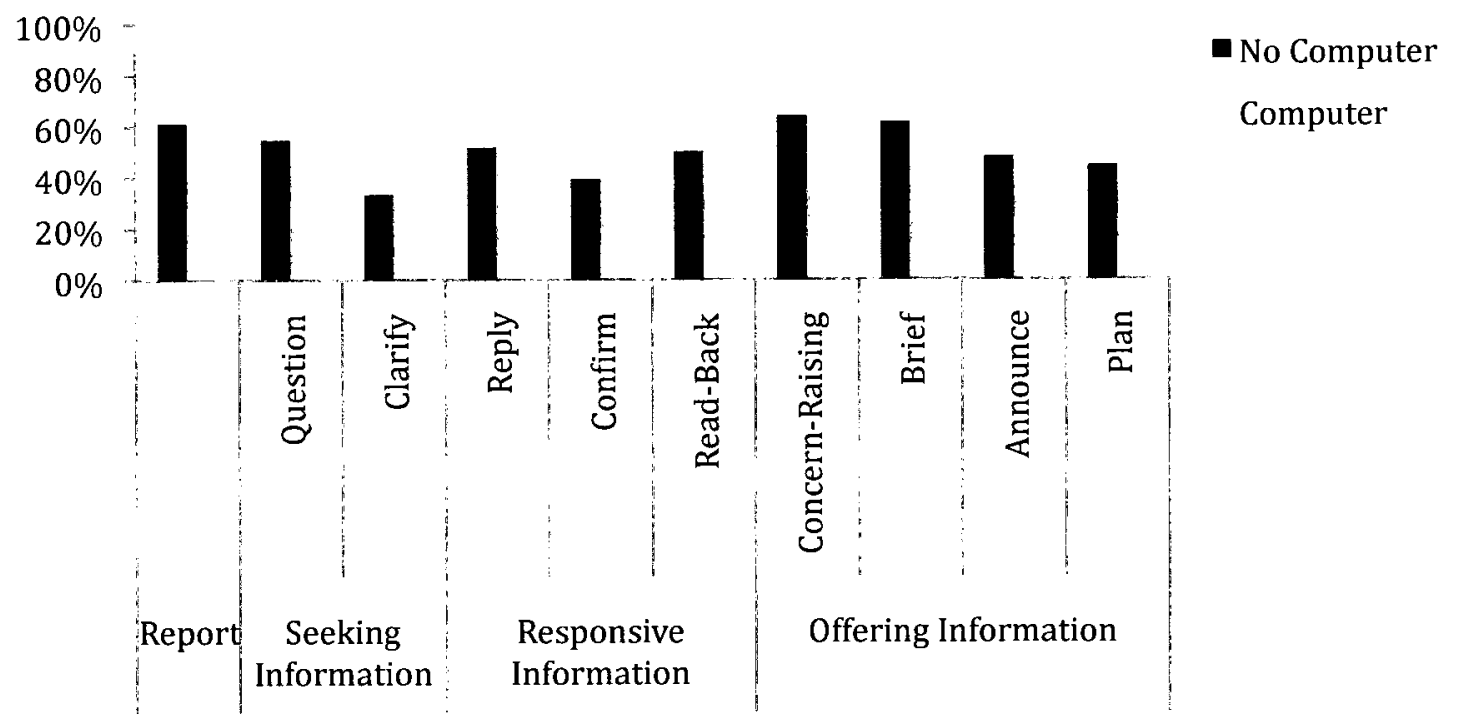

Figure 13. The frequency of verbal behaviours for handoffs without and with the use of a computer program relative to the total number of utterances for each type of verbal behaviour. 


\section{Handoff sender and receiver}

A notable difference in the positioning of primary handoff staff members (anesthetist and nurse assigned to the bed bay) involved in the exchange of information was witnessed; specifically, the physical proximity of the sender and receiver changed. The introduction of the computer program facilitated the communication to be more direct and audible to both primary parties. Anesthetists seemed to be more likely to position themselves directly beside the primary nurse, perhaps because the computer visibly identified the nurse responsible for recording the report of information. With the use of the computer program, 93\% (14 out of 15) of handoffs involved information exchange between 1 sender and 1 receiver, comparable to the absence of the program where $42 \%$ (10 out of 24) of handoffs involved this more direct method of information exchange. (It is worth nothing that out of those 10 , in five of the handoffs there was only one nurse present for the anesthetist to report to.)

\section{Prospective vs. retrospective information}

Since prospective information has been demonstrated in research to benefit the retention of information more successfully than retrospective information (Kihlgren et al., 1992; Luikkonen, 1993, \& McMahon, 1990), an analysis was run in order to investigate the differences in planning and briefing behaviours with and without use of the computer program (see Figure 14). The instances of planning with the use of the computer program (56\% of planning utterances) as a handoff tool were not significantly higher than without (44\% of planning utterances), $\chi^{2}(1, N=93)=2.44, p>.05$. There was no significant relationship found in briefing between handoffs that involved use of the computer and 
those that did not (see Figure 14). Although according to the counts of planning instances, it appeared that the computer program did facilitate planning behavior.

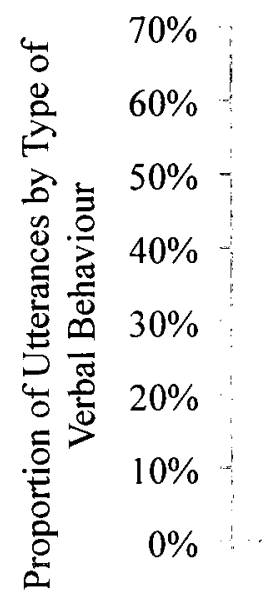

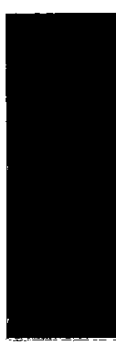

No Computer

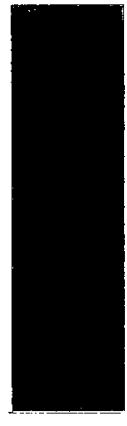

Computer $\square$ Plan

Brief

Verbal Behaviour Type

Figure 14. Proportion of planning and briefing relative to the total number of utterances observed in each verbal behavior type.

Furthermore, there was a significant relationship, $\chi^{2}(12, N=15)=23.33, p<.05$, between briefing and planning with the use of a computer versus a non-significant relationship, $\chi^{2}(15, N=24)=14.51, p<.05$, without the use of a computer. This result indicates a possible reciprocal relationship between planning and briefing with the use of a computer.

Summary of the Findings

Anesthetists used a common chunking strategy when they presented patient-relevant information in handoffs. The embedding of sub-categories of medical information was a recurring structure throughout the observational data. Although anesthetists and nurses provided the researcher with content categories that should be discussed in a handoff, the 
data from observations suggest a gap between what was suggested and what was actually reported. Four information categories emerged as the most common topics of conversation and they were: history, intra-operative events, patient status, and future care plan. These four general categories were then used to compare the types of verbal behaviours in each of those categories while incorporating an analysis of the associated speaker. The results demonstrated that reporting information was the most common anesthetist verbal behaviour and was more frequent in the history and intra-operative events categories. Seeking information was the most common nurse verbal behaviour type and was more frequent in the patient status and future care plan categories, thereby suggesting a relationship between the instances of reporting and questions. The analysis of the use of resources identified an increased instance of planning with the use of the new computer program versus without. In contrast, the instance of briefs decreased with the use of the program in comparison to that documented without the computer. The computer also appeared to promote other proactive verbal behaviours such as confirming and clarifying. The implications of these relationships will be discussed next.

\section{Discussion}

\section{General Overview}

This study was aimed at naturalistically exploring patient care handoffs with the intention of uncovering the structures and procedures for handoffs in the recovery room of an Ontario hospital. Through the use of ethnographic observations, interviews, and questionnaires, the researcher documented and analyzed communication patterns from multi-disciplinary healthcare teams. The observed communication content was also compared to a benchmark of suggested content that was obtained from interview data. 
The results are interpreted relative to the distributed cognition theoretical framework along with the future implications to patient safety and information sharing in handoffs.

\section{Handoff Structure}

\section{Observed structures}

Through analysis of the observational data a common information structure emerged. Various sub-categories exhibited recurring order within their associated general categories; in other words, the embedding of information was a common practice among handoffs. Although the order of presentation of general categories was not consistent in the observed handoffs, the fact that anesthetists tended to group sub-categories of medical information similarly may indicate a common information structure pertaining to the types of information necessary in a handoff from the OR to the recovery room.

Despite a common structure in the content, an absence of order in the presentation of information may be reflective of a lack of an overarching structure. This could be a result of the non-standardized procedure for handoffs. According to interview data, the handoff training procedure for anesthetists is limited to "see one, practice one, do one," (direct quote from interviews) where residents learn what pieces of information to provide primarily through observation. Research has cited the communication behaviours of healthcare staff as a cause of interruptions (Patel et al., 2008). When compounded with the lack of standardized protocol, this could impact susceptibility to communication breakdowns if external sources (such as interruptions) disrupt the anesthetist's report of information (Patterson et al., 2004). 


\section{Suggested structures}

According to the benchmark comparison, there was a gap between what was suggested should be conveyed in a PACU handoff and what was actually conveyed. Another notable finding within this analysis was the fact that the complexity of postoperative care was not related to the variety or total number of suggested topics discussed. These two findings could be a reflection of the case-by-case differences in patient relevant information, as it was mentioned in interviews that if there were nothing to report concerning a piece of information, it would not be presented. (The researcher was unable to question anesthetists as to whether or not a certain suggested piece of information that was not presented verbally was relevant to the handoff under observation.) However, by simply omitting certain information categories, staff on the receiving end might not be clear on whether it was purposely omitted or not.

The history information category had the highest match score when comparing suggested and observed information categories $(M=1.6$ out of a possible high score of 2$)$. In this category, the topics of allergies and comorbidities were the two sub-categories and if there was no information on these two topics it was commonplace to explicitly verbalize that there was nothing to report, e.g. "no allergies" or "otherwise healthy". Interestingly, the history category also had the lowest observed instance of questions. This demonstrates the benefit of verbalizing (to some extent) all of the important pieces of medical information that would be relevant to the receiving nurse. It also lends support to the implementation of standardization in the way information content is presented by anesthetists in order to minimize ambiguities concerning patient relevant details. 
Verbal Behaviours and Speaker

\section{Reporting and questions}

The two most common reasons for healthcare staff to communicate were to report information and seek information through question and reply. The rate of responses in relation to the number of questions posed demonstrated a positive pattern of closed-loop communication that allowed speakers to be aware of the status of their message. Since nurse utterances comprised the majority of questions and anesthetist reply rates matched that of questions by nurses, it is plausible to conclude that in the PACU, the two professions have a reciprocal relationship that opens the lines of communication. This pattern of communication also limits the potential for information loss.

\section{Handoffs Without and With Use of the Computer Program}

\section{Retrospective versus prospective information sharing}

According to a comparison of the instances of plans and briefs without and with the use of the new computer program (Periop Manager), the use of the computer may have facilitated planning behaviour. This is a beneficial effect considering past research has demonstrated that prospective information can foster improved information retention as well as promote the development of patient care plans (Kihlgren et al., 1992; Luikkonen, 1993, \& McMahon, 1990). Furthermore, briefing, which was found to be more common in handoffs without the use of the computer, can often involve redundant information. Research has revealed that briefs often include patient details that can be found in the patient chart (Sexton et al., 2004) and therefore, a focus on prospective information sharing would help nurses in obtaining information that would otherwise not be recorded. 
There was also a significant positive relationship between planning and briefing within handoffs that involved the use of the computer program. Although briefing can involve redundant information (Sexton et al., 2004), it can also be an important aspect of a PACU handoff, as certain pieces of medical information documented in the chart are pertinent and need to be presented verbally to the receiving nurse(s) so that he/she can treat the patient immediately (interview data). Once the brief has been given, the nurse(s) and/or anesthetists can then work towards a care plan that addresses the patient's needs. (This relationship was not present in the handoffs that did not involve use of the computer program.)

\section{Information content and Periop Manager}

The implementation of Periop Manager in the PACU was not associated with a notable change in the content or means of information exchange. In comparison to handoffs that did not involve the use of the computer program, on average the number of different information categories discussed did not vary and the average number of verbal behaviours only increased by two with the introduction of the computer. Furthermore, the program does not appear to strongly influence the tendency of nurses to request more of the suggested information categories (from interviews), as the overall match score only increased on average by one. The average duration along with the average number of utterances in handoffs did not differ, most likely as a result of the consistency in the characteristics of communication over the course of all of the handoff observations.

The program itself does not have mandatory fields of information input, which lends itself to the results documented above. There are "expected" fields of completion but it is ultimately at the PACU nurse's discretion to decide which information pieces to record 
onto the computer. The lack of standardization with regards to the information content in this program could lead to missing information for receiving healthcare staff at the next point of care transfer (e.g. the surgical unit).

\section{Focus on Retrospective Information Sharing}

The four most common information categories discussed in observed handoffs were history, intra-operative events, patient status, and future care plan. Of those categories, history and intra-operative events are primarily comprised of retrospective patient information and only future care plan is primarily prospectively oriented. Reporting by anesthetists was more common for the two retrospective categories and decreased proportionately in the future care plan category (see Figure 7). This suggests that a large proportion of communication in handoffs in the PACU is focused on sharing past information. This result replicates past research within various healthcare environments (Kihlgren et al., 1992; Luikkonen, 1993; McMahon, 1990). The implication of this finding is that if any fields in Periop Manager were to be made mandatory, it would be beneficial to make information concerning future care plans (medications, treatments, discharge, etc.) a recording standard since anesthetists are already proactively offering retrospective medical information. This suggestion is further supported by Dowding's research (2001), which found that nurses were more likely to recall prospective information when receiving a verbal report of patient information during end of shift handoffs. 
Study Implications

\section{Theoretical implications}

In PACU handoffs, tasks concerning the communication of patient-relevant details were primarily distributed between individual health care providers and the team. Verbal communications emerged as the major channel of information exchange between multidisciplinary care providers, although references to artifacts such as computers were also a fundamental aspect of recording important handoff information. Empirical evidence supports this finding as past studies have also documented verbal behaviour as the main source of information exchange within a hospital environment (Coiera, 2006; Parker \& Coiera, 2000). The analysis of communication patterns, specifically the verbal behaviours, revealed several characteristics of PACU handoffs.

The workflow in the PACU was grounded by the social nature of the environment, which was evidenced by the fact that all of the observed handoffs were verbally interactive; this is a similar finding to that of a previous study that investigated distributed cognition within the context of an emergency co-ordination center (Artman \& Waern, 1999). Verbalized information instigated the use of tools because within the handoff context, paper forms (e.g. the Anesthesia Record Sheet was used for every handoff) and the computer program were primarily used as a means to transform the information so that it could be referenced at a later point in time. Thus, the artifacts in the PACU may contribute to the potential for information to be shared in the future (Artman \& Garbis, 1998). The task of conveying patient details was clearly distributed across the system as a large proportion of information propagated from one individual (anesthetist) in the form of a verbal report to another individual (receiving nurse), who then transformed the 
information by recording it on paper or the computer program. In theoretical terms, the information was carried from internal representations in the mind of individuals, to external representations in the form of documentation (Hazelhurst, McMullen, \& Gorman, 2007).

Nurses in the PACU work in an environment where they are almost always expected to construct a detailed understanding of the situation through indirect information. Within this specific healthcare context where information sharing is a core component, the importance of teamwork is emphasized. A complete picture of the situation cannot be obtained individually, which is why distributed cognition is appropriate for analyzing these dynamic environments (Artman \& Garbis, 1999; Hazelhurst, McMullen, \& Gorman, 2007; Hollan et al., 2000; Rogers \& Ellis, 1994). Indications of the element of collaborative teamwork in PACU handoffs was evidenced by the verbal behaviours employed to seek information and respond to information requests. These behaviours demonstrated that there is a strong interactive component to a handoff. The matched rate of responses to questions speaks to the positive pattern of collaborative information transfer across the team. Furthermore, the link between the decline in the instance of reporting and the increase in the instance of questions demonstrates that communication events are inter-related over the duration of the handoff. This result could also be indicative of an attempt on behalf of the nurses to co-ordinate the knowledge and resources that will be required for the patient's care (Artman \& Garbis, 1998). Research has demonstrated that in situations involving multiple healthcare professions, individual information requirements can vary (The Royal College of Surgeons, 2007). In the PACU, handoffs always involve a multi-disciplinary dyad, which is why it is necessary for 
healthcare staff to work as a team to constantly update each other and share individual knowledge in order to minimize the potential for information loss.

One of the goals of the present thesis was to provide preliminary suggestions that may be useful for the future design of support tools for handoffs. By documenting the characteristics of handoffs through naturalistic observation, the researcher was able to understand the existing processes (multi-disciplinary team, information flow, content of communication) surrounding PACU handoffs. Understanding the processes surrounding handoffs is the first step towards investigating the benefits of newly introduced information technology, such as Periop Manager (Hazelhurst, McMullen, \& Gorman, 2007; Stead et al., 2004). The interaction between nurses and the new computer program appeared to benefit patient care because of the documented increase in the frequency of prospective verbal behaviours. This may seem to be of minimal significance however, this result hints at the potential for improvements through the use of information technology. Although it is acknowledged that the program is in the preliminary stages of implementation and will continue to evolve, it can be noted that currently the program is not utilized to its full potential. This is because it is primarily used as a recorder rather than an interactive tool that can aid the process. Specifically, Periop Manager has the potential to provide cues for collecting valuable information. This would be achievable through the implementation of mandatory completion fields.

\section{Implications to patient safety}

Past research has shown that in order for clinicians to feel comfortable voicing their concerns and questions there has to be an explicit safety culture present (Leonard et al., 2004). The results of the present thesis point towards the presence of such a culture as 
nurses posed a fair number of questions that were matched by anesthetists and fellow nurse replies. Interview data also pointed at the existence of a mutual respect between the handoff team. Anesthetists listed "confirming with the nurse" as one of the requirements for a verbal handoff to ensure the nurses' satisfaction with the handoff; this can in turn affect the safety of each patient's care in the recovery room. The "power distance" referred to in the literature (Donchin et al., 1995; Williams et al., 2007) did not appear to be a barrier to effective communication within the context of the PACU. The volume of verbal behaviours and discussions concerning patient details speaks to the open lines of communication between physicians and nurses in this specific hospital environment.

Within the context of the recovery room, nurses are experienced and are comfortable enough with surgically related handoffs to know which questions to ask in order to fill in any gaps left by anesthetists. However, this may not always be the case in other hospital units where verbal handoffs are not so common and nurses are not as experienced. Although the rate of replies matched that of questions, overall, the high frequency of questions especially in the future care plan information category, indicates that nurses are not getting all of the information upfront that they require to care for the patient. A standard for verbalized information by anesthetists should be implemented for information concerning current patient details including tasks to be done with the patient immediately upon their arrival in the PACU, as well as for their future care plan.

Anesthetists conveyed in interviews that if certain pieces of information were not applicable to the specific case, e.g. there was no blood loss to report, the entire topic would be omitted from the handoff. However, analysis of suggested information categories for the history category revealed that when information was not applicable, 
anesthetists explicitly mentioned that there was nothing to report on the subject.

Consequently, there was a very small proportion of questions in this information category. The research would suggest that by offering general information regardless of the presence of specific details pertaining to the topic, anesthetists could help to minimize the potential for perceived information loss. This should be practiced as a standard of information exchange during handoffs.

\section{Study Limitations}

There were several limitations involved in the research; the majority of limits were a direct result of the context of study. Due to the hospital's requirement to consent patients pre-observation, in addition to the limited number of patients whose care would provide a point of observation, the researcher was unable to pre-select handoffs based on the specific anesthetist assigned to the case. Therefore, several observations involved the same anesthetist giving the report and the assumption of independence of sampling was violated. Small staff numbers would also not have allowed for the observation of different people every handoff. The limited number of staff members and the constraints on their time due to the intensity of the working environment also lead to small sample sizes for the interview data, which minimized the ability to capture the ideal depth of data on the variables of interest.

A limitation was placed on the overall generalizability of the research findings because observations were completed in one hospital unit only. Although approval was granted in a surgical unit, the organizational structure of the recovery room meant that patients could come out of the OR with very short notice and therefore, in order to capture the handoff, the researcher had to remain in the PACU. It is possible that 
different perioperative hospital units might require different types of patient -relevant handoff information in order to provide the appropriate care.

Finally, there was only one researcher present to document the details of each handoff and as a result certain details, such as interruptions, were not captured. Future research in this area would benefit from recording the various types and levels of interruptions during communication exchanges in handoffs. These behaviours could influence the breadth of information that is conveyed. Interruptions have also been documented as a major contributor to information loss that in turn has the potential to endanger patient safety (Delucia, Ott, \& Palmieri, 2009; McGillis, Pederson, \& Fairley, 2010).

Despite the limitations, the findings are representative of other similar hospital settings. The observed patterns of communication and the impact of the use of resources on healthcare providers can be generalized to various hospital units, as these results would most likely be common to other types of multidisciplinary care teams.

Furthermore, previous in-hospital communication research (Foster-Hunt, 2009; Kramer, 2009; Parush et al., 2009) has observed similar scenarios and produced useful findings that have been applicable across healthcare settings.

\section{Future Research Directions}

The finding that anesthetists use a common chunking strategy when presenting medical information in handoffs supports further investigations into the communication strategies employed in these types of exchanges. Verbal communication as the major channel of information exchange confirms the necessity for standardization to the content of handoffs. The analysis revealed that anesthetists in the PACU are more likely to proactively provide retrospective information and therefore, future research should 
investigate the benefits of requiring prospective information to be provided. The documentation of the practices and procedures for handoffs supports the advancement of research in this field, especially into the effects of introducing technology as a method for improving the efficiency of handoffs.

There is evidence from past research in the healthcare field that even the standardization of verbal communication does not necessarily improve the recall of information (Talbot \& Bleetman, 2007), therefore, it is important for future research to continue to elucidate how technological artifacts can supplement the handoff. A recent study that employed a similar methodology and context to the present thesis also uncovered informal processes for information exchange during handoffs in a recovery room (Smith, Pope, Goodwin, \& Mort, 2008). There is an opportunity for information retention to be improved with the implementation of standards by means of information technology.

Past research on recovery room handoffs has tended to focus on investigating whether certain details were verbally presented, without placing a focus on documenting the regular practices and procedures for transferring information (Smith et al., 2008). In order for future research to investigate contextual factors (such as interruptions) that can lead to communication breakdowns and information loss, it is first important to understand the nature of the environment under study. Future research endeavors can use the procedures and communication patterns documented in the present thesis to build upon and in turn, develop concrete suggestions and design criteria for the development of handoff support tools. 
References

Alverado, K., Lee, R., Christofferson, E., Fram, N., Boblin, S., Poole, N., et al. (2006). Transfer of accountability: Transforming shift handover to enhance patient safety. Healthcare Quarterly, 9, 75-79.

Alvarez, G., \& Coiera, E. (2006). Interdisciplinary communication: an uncharted source of medical error? Journal of Critical Care, 21, 236-242.

Artman, H., \& Garbis, C. (1998). Situation awareness as distributed cognition. In T. Green, L. Bannon, C. Warren, Buckley (Eds.), Cognition and cooperation (pp. 151156). Proceedings of 9 th Conference of Cognitive Ergonomics.

Ayse, P., \& Gurses, X. Y. (2006). A systematic review of the literature on multidisciplinary rounds to design information technology. Journal of the American Medical Informatics Association, 13(3), 267-276.

Baddeley, A.D., Thomson, N., \& Buchanan, M. (1975). Word length and the structure of short-term memory. Journal of Verbal Learning and Verbal Behaviour, 14, 575-589.

Baker, D.P., Day, R., \& Salas, E. (2006). Teamwork as an essential component of highreliability organizations. Health Research and Educational Trust, 41(4), 1576-1598.

Beckman, U., Gillies, D.M., Berenholtz, S.M., Wu, A.W., \& Provost, P. (2004). Incidents relating to the intra-hospital transfer of critically ill patients. Intensive Care Medicine, $30,1579-1585$.

Bell, C.M., Schnipper, J.L., Auerbach, A.D., Kaboli, P.J., Wetterneck, T.B., Gonzales, D.V., et al. (2008). Association of communication between hospital-based physicians and primary care providers with patient outcomes. Journal of General Internal Medicine, 24(3), 381-386. 
Blandford, A., \& Furniss, D. (2006). DiCoT: a methodology for applying distributed cognition to the design of team working systems. In S. Gilroy \& M. Harrison (Eds.), Interactive Systems, Design, Specification, and Verification, (pp. 26-38). Springer, DOI.

Bowers, H.F. (2006). Designing quality course management systems that foster intraprofessional education. Nurse Education Today, 26, 726-731.

Carayon, P., Hundt, A., Alvarado, C.J., Springman, S.R., \& Ayoub, P. (2006). Patient safety in outpatient surgery: the viewpoint of the healthcare providers. Ergonomics, $49(5,6), 470-480$.

Christian, C.K., Gustafson, M.L., Roth, E.M., Sheridan, T.B., Gandhi, T.K., Dwyer, K., et al. (2006). A prospective study of patient safety in the operating room. Surgery, $139,159-173$.

Coiera, E., \& Tombs, V. (1998). Communication behaviours in a hospital setting: an observational study. British Medical Journal, 316, 673-677.

Covell D.G., Uman G.C., \& Manning P.R. (1985). Information needs in office practice: are they being met? Annals of Internal Medicine, 130, 596-599.

Donchin, Y., Gopher, D., Olin, M., Badihi, Y., Biesky, M., \& Sprung, C.L. (1995). A look into the nature and causes of human errors in the intensive care unit. Critical Care Medicine, 23(2), 294-300.

Dowding, D. (2001). Examining the effects that manipulating information given in the change of shift report has on nurses' care planning ability. Journal of Advanced Nursing, 33(6), 836-846. 
Dykes, P.C., Hurley, A., Cashen, M., Bakken, S., \& Duffy, M.E. (2007). Development and psychometric evaluation of the impact of health information technology (I-HIT) scale. Journal of the American Medical Informatics Association, 14(4), 507-514.

Eggland, E. T., \& Heinemann, D. S. (1994). The reporting process. In D.L. Hilton \& B. Ryalls (Eds.), Nursing Documentation (pp. 209-223).

Foster-Hunt, T. (2009). Information flow in change of shift patient handoffs in a pediatric intensive care unit. Unpublished master's thesis, Carleton University, Ottawa, Ontario, Canada.

Greenberg, C.C., Regenbogen, S.E., Studdert, D.M., Lipsitz, S.R., Rogers, S.O., Zinner, M.J., et al. (2007). Patterns of communication breakdowns resulting in injury to surgical patients. American College of Surgeons, 533-540.

Halverson, C.A. (1994). Distributed cognition as a theoretical framework for HCI: don't throw the baby out with the bathwater - the importance of the cursor in air traffic control. Unpublished position paper, University of California, San Diego, USA. Hazelhurst,B., McMullen, C.K., \& Gorman, P.N. (2007). Distributed cognition in the heart room: how situation awareness arises from coordinated communications during cardiac surgery. Journal of Biomedical Informatics, 40, 539-551.

Helmreich, R.L. (2000). On error management: lessons from aviation. British Medical Journal, 320, 781-785.

Hollan, J., Hutchins, E., \& Kirsh, D. (2000). Distributed cognition: toward a new foundation for human-computer interaction research. ACM Transactions on Computer-Human Interaction, 7, 174-196. 
Horwitz, L. I., Meredith, T., Schuur, J.D., Shah, N.R., Kulkarni, R., \& Grace, Y. (2009). Dropping the baton: a qualitative analysis of failures during the transition from emergency department to inpatient care. Annals of Emergency Medicine, 53(6), 701710.

Hutchins, E. (2003). Cognitive ethnography. Plenary address at the 25th meeting of the Cognitive Science Society, Cambridge, MA.

Kachalia, A., Gandi, T.K., Puopolo, A.L., Yoon, C., Thomas, E.J., Griffey, R., et al. (2007). Missed and delayed diagnoses in emergency department: a study of close malpractice claims from 4 liability insurers. Annals of Emergency Medicine, 49(2), $196-205$.

Kerr, M. P. (2002). A qualitative study of shift handover practice and function from a socio-technical perspective. [Electronic version] Journal of Advanced Nursing, 37, $125-135$.

Kaafarani, H., Itani, K., Rosen, A., Zhao, S., Hartman, C., \& Gaba, D. (2009). How does patient safety culture in the operating room and post-anesthetic care unit compare to the rest of the hospital? The American Journal of Surgery, 198, 70-75.

Kilgren, M., Lindsten, I.G., Norberg, A., \& Karlsson, I. (1992). The content of the oral daily reports at a long-term ward before and after nurses training in integrity promoting care. Scandinavian Journal of Caring Science, 6, 105-112.

Kohn, L.T., Corrigan, J.M., \& Donaldson, M.S. (2000). To err is human: Building a safer health system. Washington, DC: National Academy Press. 
Kramer, C. (2009). Communication linking team mental models and team situation awareness in the operating room. Unpublished master's thesis, Carleton University, Ottawa, Ontario, Canada.

Kramer, C., Parush, A., Brandigampola, S., \& Momtahan, K. (2010). Analysis of crossprofessional communication in thoracic operating rooms. In Vincent G. Duffy (Ed.), Advances in Human Factors and Ergonomics (pp. 307-316). CRC Press.

Leonard, M., Graham, S., \& Bonacum, D. (2004). The human factor: the critical importance of effective teamwork and communication in providing safe care. Quality and Safety in Healthcare, 13, 85-90.

Lingard, L., Espin, S., \& Whyte, S., Regehr, G., Baker, G.R., Reznick, R., et al. (2004). Communication failures in the operating room: an observational classification of recurrent types and effects. Quality and Safety in Healthcare, 13, 330-334.

Luikkonen, A. (1993). The content of nurses' oral shift reports in homes for elderly people. Journal of Advanced Nursing, 18, 1095-1100.

McMahon, R. (1990). What are we saying? Nursing Times, 86, 38-40.

Norsen, L., Opladen, J., \& Quinn, J. (1995). Practice model: collaborative practice. Critical Care Nursing Clinics of North America, 7, 43-52.

Odom-Forren, J. (2007). Accurate patient handoffs: imperative for patient safety. Journal of Perianesthesia Nursing, 22(4), 233-234.

Oulasvirta, A., \& Saariluoma, P. (2006). Surviving task interruptions: investigating the implications of long-term working memory theory. International Journal of HumanComputer Studies, 64, 941-961. 
O'Byrne, W.T., Weavind, L., \& Selby, J. (2008). The science and economics of improving clinical communication. Anesthesiology Clinics, 26, 729-744.

Patel, V.L., Zhang, J., Yoskowitz, N.A., Green, R., \& Sayan, O.R. (2008). Translational cognition for decision support in critical care environments: a review. Journal of Biomedical Informatics, 41(3), 413-431.

Patterson, E.S., Roth, E.M., Woods, D.D., Chow, R., \& Orlando Gomes, J. (2004). Handoff strategies in settings with high consequences for failure: lessons learned for health care operations. The International Journal on Quality of Health Care, 16(2), $125-132$.

Parker, J., \& Coiera, E. (2000). Improving clinical communication. Journal of American Medical Informatics Association, 7(5), 453-461.

Parush, A., Kramer, C., Foster-Hunt, T., Momtahan, K., Hunter, A., \& Sohmer, B. (in press). Communication indicators for team situation awareness in the cardiac OR: implications for augmentative technology. Journal of Biomedical Informatics.

Parush, A., Momtahan, K., Foster-Hunt, T., Kramer, C., Hunter, A., \& Howard, N. (2009). A communication analysis methodology for developing a cardiac operating room team-oriented display. Proceedings of the Human Factors and Ergonomics Society $53^{\text {rd }}$ Annual Meeting, 728-732.

Pesanka, D.A., Greenhouse, P.K., Rack, L.L., Delucia, G.A., Perret, R.W., Scholle, C.C., et al. (2008). Ticket to ride: reducing handoff risk during hospital patient transport. Journal of Nursing Care Quality, 24(2), 109-115.

Reitman, J.S. (1974). Without surreptitious rehearsal, information in short-term memory decays. Journal of Verbal Learning and Verbal Behaviour, 13, 365-377. 
Rich, V., Garcia Sullivan, P., \& Kirby, A. (2007). Pioneers in patient flow improvement: combining people, processes, and technology. Nurse Leader, 52-56.

Ross, J. \& Ranum, D. (2009). Improving patient safety by understanding past experiences in day surgery and PACU. Journal of Perianesthesia Nursing, 241(3), 144-51.

Safran C., Sands D.Z., \& Rind D.M. (1999). Online Medical Records: a decade of experience. Methods of Information in Medicine, 38(4, 5), 308-312.

Sandalowski, M. (1995). Sample size in qualitative research. Research in Nursing and Health, 18, 179-183.

Sexton, A., Chan, C., Elliott, M., Stuart, J., Jayasuriy, R., \& Crookes, P. (2004). Nursing handovers: do we really need them? Journal of Nursing Management, 12, 37-42.

Shultz, K., Carayon, P., Schoofs Hundt, A., \& Springman, S.R. (2007). Care transitions in the outpatient surgery preoperative process: facilitators and obstacles to information flow and their consequences. Cognitive Technology Work, 9, 219-231.

Simoneau, Y. (2009). The use of resources during shift reports in a pediatric intensive care unit. Unpublished undergraduate honours thesis, Carleton University, Ottawa, Ontario, Canada.

Singh, H., Aanand, D.N., Raghuram, R., \& Peterson, L.A. (2007). Reducing diagnostic errors through effective communication: harnessing the power of information technology. Journal of General Internal Medicine, 23(4), 489-494.

Smith, A.F., Pope, C., Goodwin, D., \& Mort, M. (2008). Interprofessional handover and patient safety in anesthesia: observational study of handovers in the recovery room. British Journal of Anesthesia, 101, 332-337. 
Solet, D.J., Norvell, J.M., Rutan, G., \& Frankel, R. (2005). Lost in translation: challenges and opportunities in physician-to-physician communication during patient handoffs. Academic Medicine, 80(2), 1094-1099.

Stead, W.W., Haynes, R.B., Fuller, S., Friedman, C.P., Travis, L.E., Beck, J.R., et al. (2004). Designing medical informatics research and library-resource projects to increase what is learned. Journal of American Medical Informatics Association, 1(1), 28-33.

Strauss, A., \& Corbin, J. (1990). Basics of qualitative research. London: Sage.

The Joint Commission. National patient safety goals--2008. Retrieved June 15, 2009, from http://www.jointcommission.org/NR/rdonlyres/31666E86-E7F4-423E-9BE8F05BD1CB0AA8/0/HAP_NPSG.pdf.

Talbot, R., \& Bleetman, A. (2007). Retention of information by emergency department staff at ambulance handover: do standardized approaches work? Emergency Medicine Journal, 24, 539-42.

The World Health Organization (2008). 10 facts on patient safety. Retrieved May 2009 Retrieved from: http://www.who.int/features/factfiles/patient_safety/en/index.html Varkey, P., Rose, S., \& Swensen, S. (2009). A patient safety curriculum for graduate medical education: results from a needs assessment of educators and patient safety experts. American Journal of Medical Quality, 24(3), 214-221.

Webster, J. (1999). Practitioner-centered research: an evaluation of the implementation of the bedside handover. [Electronic version] Journal of Advanced Nursing, 30, 13751382. 
Williams, R.G., Silverman, R., Schwind, C., Fortune, J.B., Sutyak, J., Horvath, K.D., et al. (2007). Surgeon information transfer and communication, factors affecting quality and efficiency of inpatient care. Annals of Surgery, 245(2), 159-169.

Wilson, R.M., Runciman, W.B., Gibberd, R.W., Harrison, T., Newby, L., \& Hamilton, J.D. (1995). The quality in Australian health care study. Medical Journal of Australia, $163,458-471$.

Xyrichis, A., \& Lowton, K. (2008). What fosters or prevents interprofessional teamworking in primary and community care? A literature review. International Journal of Nursing Studies, 45, 140-153.

Yeager, S. (2005). Interdisciplinary collaboration: the heart and soul of health care. Critical Care Nursing, 17, 143-148.

Zwarenstein, M., Reeves, S., Russell, A., Kenaszchuk, C., Gotlib Conn, L., Miller, K.L., et al. (2007). Structuring communication relationships for inter-professional teamwork (SCRIPT): a cluster randomized controlled trial. Trials, 8(23), 1-14. 
Appendix A

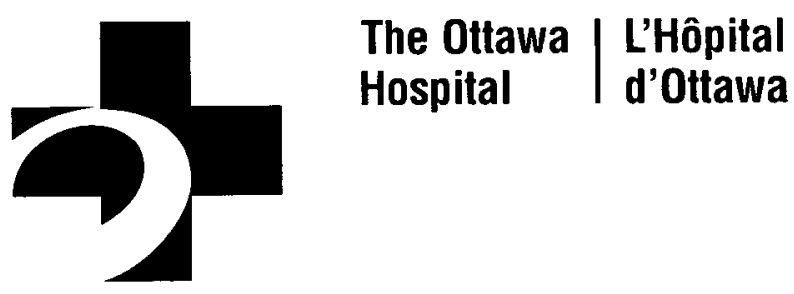

\section{PATIENT INFORMATION SHEET AND CONSENT}

\section{Communication in Patient Care Handoffs and Implications to Safety}

INVESTIGATOR: Avi Parush, PhD 613-520-2600 ext 6026

INVESTIGATOR: Kathryn Momtahan, RN, PhD 613-798-5555 ext 16035

CO-INVESTIGATOR: Linda Hunter, RN, MScN 613-798-5555 ext 16695

CO-INVESTIGATOR: Pam Bush, RN, BScN 613-737-8899 ext 72797

MASTER'S STUDENT: Alicia McMullan 613-520-2600 ext 6026

Please read this Participant Information Sheet and Consent Form carefully and ask as many questions as you like before deciding whether to participate.

\section{INTRODUCTION}

The goal of the present research is to promote the culture of patient safety by avoiding future preventable medical errors that can result from a loss of information during patient transfers. Patient transfers occur, for instance, between the operating room and the recovery room. Communication is a core component of patient care transitions when patients move from one area of the hospital to another. The purpose of the proposed research is to study current practices and procedures during patient handoffs. In the short term, we aim to improve the understanding of current handoff procedures and their susceptibility to loss of information. Data collected from this study will also be used for the purpose of the long term goal of contributing to the development of safe, effective, and efficient policies and procedures and to propose design criteria for the development of tools for use in handoffs such as checklists and/or computing devices.

\section{PROCEDURE}

One or two members of the research team will observe and take notes on the communication patterns and use of equipment for the transfer of information among the medical team involved in patient care handoffs between the operating rooms and the post anesthetic recovery room.

\section{RISKS and DISCOMFORTS of PARTICIPATION}


There are no anticipated risks or discomforts involved in this study.

\section{BENEFITS OF PARTICIPATION}

There are no direct benefits of this study to you personally. However, it is hoped that the information gathered will benefit future patients of The Ottawa Hospital through the minimization of potential medical errors by gaining a deeper understanding of the patient handoff process. There is no monetary compensation to being in the study.

\section{CONFIDENTIALITY}

All records will be kept confidential. You will not be identified in any publications by name or initials. All data sheets will have your independent study number only on them. They will be kept in a locked cabinet in a locked office at Carleton University while data is being analyzed and will then be kept in a locked cabinet in a locked office at The Ottawa Hospital and will be disposed of after a period of 15 years. Original patient consent forms will be kept in a locked cabinet in a locked office at The Ottawa Hospital, also for a period of 15 years. Any information gathered on computers will be kept on a password protected secure network at Carleton University while data is being analyzed and will then be kept on the password protected secure network at The Ottawa Hospital and will be deleted after a period of 15 years.

\section{TERMINATION OF THE STUDY}

Your participation is voluntary, and you may choose not to participate or to discontinue participation at any time.

\section{ETHICS}

This study has been approved by The Ottawa Hospital Research Ethics Boards. The Board considers the ethical aspects of all research projects at The Ottawa Hospital using human participants. If you have any questions about your rights as a research participant, you may contact the Chairman of The Ottawa Hospital Research Ethics Boards at 613798-5555, extension 14902.

This study has also been approved by the Human Research Ethics Committee at Carleton University. If you have any questions, you may contact Dr. Monique Senechal, Chair of The Carleton University Ethics Committee for Psychological Research: 613-520-2600 ext.1155, monique senechal@carleton.ca.

\section{PARTICIPATION Participation in research is completely voluntary.}

You are free to choose whether to participate in this study or not. If you choose to participate, you may choose to withdraw your consent at any time. This will not impact your present or future care at this hospital. 


\section{Communication in Patient Care Handoffs and Implications to Safety CONSENT TO PARTICIPATE IN RESEARCH}

I understand that I am being asked to participate in a research study involving observations of equipment and staff in the transfer of my care from the operating room to the recovery room, which is the first step in developing tools and technological devices to help staff with communications in the transfer of patients within the hospital. I have read and understood this 3-page Patient Information Sheet and Consent Form. All of my questions at this time have been answered to my satisfaction. If I have any further questions about this study, I understand that I may contact the Principal Investigators Kathryn Momtahan, RN, PhD at The Ottawa Hospital at 613-798-5555, x 16035 or Avi Parush, PhD at Carleton University at 613-520 2600 ext. 6026.

I will receive a signed copy of this Consent Form and the attached Patient Information Sheet.

I voluntarily agree to participate in this study.

\section{Signatures}

Participant's Name:

Participant's Signature:

Date:

\section{Investigator Statement (or Person Explaining the Consent)}

I have carefully explained to the research participant the nature of the above research study. To the best of my knowledge, the research participant signing this consent form understands the nature, demands, risks and benefits involved in participating in this study. I acknowledge my responsibility for the care and well being of the above research participant, to respect the rights and wishes of the research participant, and to conduct the study according to applicable Good Clinical Practice guidelines and regulations.

Name of

Investigator/Delegate Signature of

Investigator/Delegate 
Appendix B

\section{Handoff Observation Tool}

Form of Communication: Verbal Written (Specify, i.e. anesthesia record sheet)

\section{Mode of Communication:}

Real-time interaction concerning patient details

Exchange of patient chart only

Real-time interaction but no patient details conveyed

\section{Staff Involved:}

One Transmitter and One Receiver

Broadcast to Multiple Staff

Multiple Sequential Breakdowns

Number of People Involved:

1234 or more Specify discipline/numbers

\section{Disciplines Involved:}

Cross-disciplinary (i.e. nurse to anesthetist) Intradisciplinary(i.e. nurse to nurse)

Information Conveyed Related To:

Preoperative information or past medical history

Intra-operative events

Patient Status

Drugs

Dosages

Events Outside of the OR 
Future Plan of Care

Missing

The Patient Was Involved in Process: _ yes no _ If yes, how:

Notes (teamwork, openness of communication, problem solving/decision making, awareness of situation and patient, communication failures, points of failure): 


\section{Appendix C}

\section{Complexity Ratings}

Please rate the following surgeries on complexity. Please choose a number according to how complex the patient's care would be post-operatively in the recovery room. *If there are no comorbidities listed here, there were none mentioned in the report.

Removal of Bladder and Creation of Neo Bladder

- Comorbidities-Coronary disease, stable Angina (treatment for Comorbidity is Stents)

$\begin{array}{llllll}1 & 2 & 3 & 4 & 5\end{array}$

(Not At All

(Highly Complex)

Complex)

TURP

- History of AF

$\begin{array}{llllll}1 & 2 & 3 & 4 & 5\end{array}$

(Not At All

(Highly Complex)

Complex)

Hemi-colectomy; Iliocecal resection, laparoscopic

$\begin{array}{llllll}1 & 2 & 3 & 4 & 5\end{array}$

(Not At All

(Highly Complex)

Complex) 
Radical Prostatectomy

$\begin{array}{lllll}1 & 2 & 3 & 4 & 5\end{array}$

(Not At All

(Highly Complex)

Complex)

Circumcision

$\begin{array}{lllll}1 & 2 & 3 & 4 & 5\end{array}$

(Not At All

(Highly Complex)

Complex)

Gal Bladder removed

$\begin{array}{lllll}1 & 2 & 3 & 4 & 5\end{array}$

(Not At All

(Highly Complex)

Complex)

Gastric Bypass

- Comorbidities- "Sig. history", diabetes, kidneys okay, severe asthma, doesn't use puffers daily, coronary artery disease, doesn't really get chest pain, "Sig. allergies."
1
2
3
4
5

(Not At All

(Highly Complex)

Complex) 
Excision of Urachal Mass, Cystectomy-Partial (unusual surgery)

- Comorbidity-colitis which is treated and stable, chronic lower leg pain (no meds for leg pain)

$\begin{array}{lllll}1 & 2 & 3 & 4 & 5\end{array}$

(Not At All

(Highly Complex)

Complex)

Anterior Urethroplasty + Penile reconstruction (Quad/Parapelegic)

- Comorbidity-Spina Bifida, quad/para, severe reaction to latex (last year's surgery)
1
2
3
4
5

(Not At All

(Highly Complex)

Complex)

Partial Gastrectomy/Laparoscopic/possible open, removal of accessory spleen

- Comorbidities-diabetes, sleep apnea, hypertension

- Past Conditions- Had prostate cancer in the past, also had a Gastric Bypass

$\begin{array}{lllll}1 & 2 & 3 & 4 & 5\end{array}$

(Not At All

(Highly Complex)

Complex) 
TURP

- Comorbidity-Raynaud's disease, arthritis, hypertension

$\begin{array}{lllll}1 & 2 & 3 & 4 & 5\end{array}$

(Not At All

(Highly Complex)

Complex)

Radical Prostatectomy

$\begin{array}{lllll}1 & 2 & 3 & 4 & 5\end{array}$

(Not At All

(Highly Complex)

Complex)

Nephrectomy-laparoscopic, Excision of penile lesion

- Comorbidity-high cholesterol

$\begin{array}{lllll}1 & 2 & 3 & 4 & 5\end{array}$

(Not At All

(Highly Complex)

Complex) 
Prostatectomy-Radical

- Comobidity- high cholesterol

$\begin{array}{lllll}1 & 2 & 3 & 4 & 5\end{array}$

(Not At All

(Highly Complex)

Complex)

Resection of Liver Mets

- Comorbidity-diabetes, hypertension.

1

2

3

4

5

(Not At All

(Highly Complex)

Complex)

Pancreatoduodenectomy

$\begin{array}{lllll}1 & 2 & 3 & 4 & 5\end{array}$

(Not At All

(Highly Complex)

Complex)

Modified Radical Mastectomy

$\begin{array}{lllll}1 & 2 & 3 & 4 & 5\end{array}$

(Not At All

(Highly Complex)

Complex 
Removal of Penile Implant, Penile Prosthesis Insertion

- Comorbidities- Angina, stroke (with speech deficit), Anxiety Disorder, Asthma, Diabetes ("Doesn't take Insulin for Diabetes but will be taking it soon."), exercise capacity severely limited due to these comorbidities

- Surgical History- "Quintuple Bypass in 1994. Previous Penile Implant revised resulted in post-op chest pain."
1
2
3
4
5

(Not At All

(Highly Complex)

Complex)

Mesh Repair of Ventral Hernia

- Comorbidities- "Hernia for about a year."

$\begin{array}{lllll}1 & 2 & 3 & 4 & 5\end{array}$

(Not At All

(Highly Complex)

Complex)

Left Mastectomy with Axillary LN Dissection

- Comorbidity-asthma

$\begin{array}{llllll}1 & 2 & 3 & 4 & 5\end{array}$

(Not At All

(Highly Complex)

Complex) 
Salvage Radical Prostatectomy

- Surgical History- "Previous surgery had a problem with the Epidural."

$\begin{array}{lllll}1 & 2 & 3 & 4 & 5\end{array}$

(Not At All

(Highly Complex)

Complex)

TURP

1

2

3

4

5

(Not At All

(Highly Complex)

Complex)

Reversal of Hartmann Colostomy

- Comorbidity- Diabetes

- Had a cold 5 days ago

$\begin{array}{lllll}1 & 2 & 3 & 4 & 5\end{array}$

(Not At All

(Highly Complex)

Complex) 
Laparascopic Liver Cyst Excision

- Comorbidities- Hypertension, Osteoporosis

$\begin{array}{llllll}1 & 2 & 3 & 4 & 5\end{array}$

(Not At All

(Highly Complex)

Complex)

Cholecystectomy/Open

- Comorbidity- CLL

$\begin{array}{lllll}1 & 2 & 3 & 4 & 5\end{array}$

(Not At All

(Highly Complex)

Complex)

Total Parathyroidectomy and Re-implantation into Right Arm

- Comorbidity- chronic renal failure ("Potassium level checked yesterday from Dialysis (3.8).")

$\begin{array}{lllll}1 & 2 & 3 & 4 & 5\end{array}$

(Not At All

(Highly Complex)

Complex) 
Carotid Endarterectomy, Vascular

- Comorbidity- history of DVTs

$\begin{array}{lllll}1 & 2 & 3 & 4 & 5\end{array}$

(Not At All

(Highly Complex)

Complex)

Left LD + Implant Right Mastopexy

- Comorbidity- Hypertension (on Metoprolol)

- TIA 15 years ago

- Allergic to Penicillin (has nausea, fainting from it)

$\begin{array}{lllll}1 & 2 & 3 & 4 & 5\end{array}$

(Not At All

(Highly Complex)

Complex)

Excision of Left Frontal Meningioma

- Comorbidity- "She has chronic ITP."

- Past Condition- Had colon cancer

$\begin{array}{lllll}1 & 2 & 3 & 4 & 5\end{array}$

(Not At All

(Highly Complex)

Complex) 


\section{Hernia Repair}

- Past Conditions: Pancreatitis, Ruptured bowel, etoh in past

- Hypertension, chronic pain

$\begin{array}{llllll}1 & 2 & 3 & 4 & 5\end{array}$

(Not At All

(Highly Complex)

Complex)

Laminectomy Lumbar

- Past Conditions: Heart attack in '99, hemorrhage.

$\begin{array}{llllll}1 & 2 & 3 & 4 & 5\end{array}$

(Not At All

(Highly Complex)

Complex)

Sigmoid Colon resection-laparoscopic, possible ovary resection

$\begin{array}{lllll}1 & 2 & 3 & 4 & 5\end{array}$

(Not At All

(Highly Complex)

Complex)

Laminectomy Lumbar

- Diabetes (Insulin dependent)

$\begin{array}{llllll}1 & 2 & 3 & 4 & 5\end{array}$

(Not At All

(Highly Complex)

Complex) 
Shoulder Total Arthroplasty Tornier Aequalis; left

- Past conditions: Numerous joints replaced

$\begin{array}{lllll}1 & 2 & 3 & 4 & 5\end{array}$

(Not At All

(Highly Complex)

Complex)

Roux-en-y Gastric Bypass Laparoscopic (BMI>40)

- Obeisity, mother diagnosed with protein deficiency

$\begin{array}{lllll}1 & 2 & 3 & 4 & 5\end{array}$

(Not At All (Highly Complex)

Complex)

GRAXIBIFEM; Axillary-Bifemoral Bypass; Left GRAXIBIFEM; Axillary-

Bifemoral Bypass; Left

- CVA, Hypertension

$\begin{array}{llllll}1 & 2 & 3 & 4 & 5\end{array}$

(Not At Al

(Highly Complex)

Complex) 
Spine Posterior Decompression/Laminectomy Lumbar

- Diabetes, Hypertension, Allergic to morphine, History of nausea and vomiting after surgery

$\begin{array}{llllll}1 & 2 & 3 & 4 & 5\end{array}$

(Not At All

(Highly Complex)

Complex)

Prostatectomy-Radical PLND

- Hypertension, allergic to Codeine

$\begin{array}{lllll}1 & 2 & 3 & 4 & 5\end{array}$

(Not At All

(Highly Complex)

Complex)

ANEVAR; EVAR; PACU overnight, difficult Intubation, Endo Table

- Coronary Artery Disease, had 1 kidney removed a year ago

$\begin{array}{lllll}1 & 2 & 3 & 4 & 5\end{array}$

(Not At All

(Highly Complex)

Complex)

Repeat arm contracture release + ulnar nerve decompression; left; Pre-op Block

$\begin{array}{lllll}1 & 2 & 3 & 4 & 5\end{array}$

(Not At All

(Highly Complex)

Complex) 


\section{Appendix D}

Questions for the Semi-Structured Interview

1. How do you perceive the safety of patient care at this hospital?

2. Describe the current procedure for a patient care handoff in this hospital.

3. What information is typically conveyed during a normal handoff?

4. What information do you think should be conveyed during a handoff?

5. Do you prefer receiving/giving (depending on which staff member if being interviewed) handoffs in a verbal or non-verbal manner?

6. What tools do you use during a handoff? 


\section{Appendix E}

Supplementary Data Tables

\section{Table 1}

Distribution of Verbal Behaviour Types Relative to the Total Number of Utterances

High Level Category

Seeking Information: $14.7 \%$
Sub-Category

Question: $12.6 \%$

Clarify: $1.9 \%$

Anticipate: $0.1 \%$

\begin{tabular}{ll}
\hline Proactive Information: $44.4 \%$ & Report: $44.4 \%$ \\
\hline Responsive Information: $20.1 \%$ & Reply: $15.6 \%$ \\
& Confirm: $3.5 \%$ \\
& Read-back: $0.9 \%$
\end{tabular}

Offering Information: $17.1 \%$

Announce: $6.0 \%$

Plan: $2.9 \%$

Concern-Raising: $1.2 \%$

Brief: $7.1 \%$

Requesting/Commanding: $1.2 \%$

For actions: $1.2 \%$

Educating: $0.3 \%$

Coach/Teach: $0.3 \%$

Commenting: $1.7 \%$

Discuss/Explain: $1.7 \%$

Irrelevant: $0.5 \%$

Socialize: $0.5 \%$ 


\section{Table 2}

Distribution of Verbal Behaviour Types by Speaker, Relative to the Total Number of

\section{Utterances}

High Level Category

Speaker

Seeking Information:

Anesthetists: $3.7 \%$

Nurses: $10.8 \%$

\begin{tabular}{|c|c|}
\hline Proactive Information/Report: & $\begin{array}{l}\text { Anesthetists: } 42.7 \% \\
\text { Nurses: } 0.3 \%\end{array}$ \\
\hline Responsive Information: & $\begin{array}{l}\text { Anesthetists: } 10.8 \% \\
\text { Nurses: } 8.9 \%\end{array}$ \\
\hline Offering Information: & $\begin{array}{l}\text { Anesthetists: } 12.1 \% \\
\text { Nurses: } 4.1 \%\end{array}$ \\
\hline Requesting/Commanding: & $\begin{array}{l}\text { Anesthetists: } 0.8 \% \\
\text { Nurses: } 0.3 \%\end{array}$ \\
\hline Educating: & $\begin{array}{l}\text { Anesthetists: } 0.3 \% \\
\text { Nurses: } 0 \%\end{array}$ \\
\hline Commenting: & $\begin{array}{l}\text { Anesthetists: } 1.0 \% \\
\text { Nurses: } 1.1 \%\end{array}$ \\
\hline Irrelevant: & $\begin{array}{l}\text { Anesthetists: } 0.5 \% \\
\text { Nurses: } 0.5 \%\end{array}$ \\
\hline
\end{tabular}


Table 3

Distribution of Verbal Behaviours Relative to the Total Number of Utterances for Each

Type

\begin{tabular}{ll} 
High Level Category & Sub-Category \\
\hline Seeking Information: & Question: \\
& No Computer: $54.7 \%$ \\
& Computer: $45.3 \%$ \\
& Clarify: \\
& No Computer: $33.3 \%$ \\
& Computer: $66.7 \%$ \\
& \\
\hline Proactive Information: & Report: \\
& No Computer: $61.1 \%$ \\
& Computer: $48.3 \%$
\end{tabular}

\begin{tabular}{ll}
\hline Responsive Information: & Reply: \\
& No Computer: $51.7 \%$ \\
& Computer: $48.3 \%$ \\
& Confirm: \\
& No Computer: $39.4 \%$ \\
& Computer: $60.6 \%$ \\
& \\
& Read-back: \\
& No Computer: $50.0 \%$ \\
& Computer: $50.0 \%$
\end{tabular}

Offering Information:

Announce:

No Computer: $48.0 \%$

Computer: $52.0 \%$

Plan:

No Computer: $44.4 \%$

Computer: $55.6 \%$

Concern-Raising:

No Computer: $64.0 \%$

Computer: $36.0 \%$

Brief:

No Computer: $61.5 \%$

Computer: $38.5 \%$ 
Table 4

Description and Examples of Content Categories Used in the Coding Process

\begin{tabular}{|c|c|}
\hline Category & Example \\
\hline Pain Management & "What should we give for pain?" \\
\hline Antibiotics & "Did the he get antibiotics in the OR?" \\
\hline Anti-emetics & $\begin{array}{l}\text { "She's feeling nauseous, what do we } \\
\text { give?" }\end{array}$ \\
\hline $\begin{array}{l}\text { Medications (other than for pain and anti- } \\
\text { nausea) }\end{array}$ & "She's on Metoprolol for hypertension." \\
\hline Drug Name & "Fentanyl" \\
\hline Drug Dose & "10 of Fentanyl." \\
\hline Drug Administration Method & "Received an injection into the wound." \\
\hline CV (Cardiovascular System) & "Is there an arterial (heart) line in?" \\
\hline Labs & "Needs a chest x-ray." \\
\hline Treatment & $\begin{array}{l}\text { "The patient got a new catheter right before } \\
\text { we brought her out." }\end{array}$ \\
\hline GU (Genitourinary System) & $\begin{array}{l}\text { "Need to keep her on the fluids for the next } \\
\text { while." }\end{array}$ \\
\hline Fluid Name & "She's currently receiving Crystaloids." \\
\hline Fluid Amount & "Give $100 \mathrm{ccs}$ over the next hour." \\
\hline Total Fluid Intake & "Two litres of fluid given in the OR." \\
\hline Blood Pressure & "Blood pressure is 112 over $64 . "$ \\
\hline Blood Loss & "She lost a litre and a half in the OR." \\
\hline Respiratory Status & "What are her respirations at?" \\
\hline Status of Lines on Patient & "She has a 16 gage on the right arm." \\
\hline Status of Patient's Body & "4 stabs on the torso." \\
\hline Allergies & "Patient is allergic to penicillin." \\
\hline Comorbidities & "He has diabetes." \\
\hline Treatment for Comorbidity & "The patient is Insulin dependent." \\
\hline Previous Condition & "Had a heart attack a year ago." \\
\hline
\end{tabular}


Communication in Patient Care Handoffs 110

\begin{tabular}{ll}
\hline SATS (Blood oxygen saturation) & "Her SATS were running good in the OR." \\
\hline Lab Result & "His Creatinine is normal." \\
\hline Anesthetic History & "Previous surgery resulted in severe nausea \\
& and vomiting." \\
\hline Time of Administration & "I gave Heparin at 9 am." \\
\hline Surgical History & "She has had almost every joint replaced. \\
& Multiple surgeries in the past." \\
\hline Lifestyle & "He is a pack-a-day smoker." \\
\hline Temperature & "She's going to need a Bear-Hugger, her \\
& temp is 35 degrees." \\
\hline
\end{tabular}




\section{Appendix F}

SBAR "Background" Report to a Physician About A Critical Situation

\begin{tabular}{lllllll} 
NEURO & CV & RESP & GI & GU & LABS & MEDS \\
\hline GSC & BP/MAP & RR & NV & U/O & Recent results & Current \\
Sedation & HR & Sp) 2 & OG/Drain & I/O & Date of last & infusions \\
ICP/CPP & ECG & Change in & output & Fluid & cultures & and rates \\
Pupils & Rhythm & ventilation or & Nutrition & balance & Blood glucose & Increasing \\
& Chest Pain & oxygenation & High & & & Inotropes \\
& Temp & Breath sounds & residuals & & & Med Error \\
& PA and cardiac & ABG & IAP & & & \\
& calcs & & Abdo pain & & \\
& CVP & & & & & \\
& Bleeding & & & & & \\
& IV access & & & & &
\end{tabular}

